\title{
Meridional variation in tropospheric methane on Titan observed with AO spectroscopy at Keck and VLT
}

\author{
Máté Ádámkovics ${ }^{\mathrm{a}}$, Jonathan L. Mitchell ${ }^{\mathrm{b}, \mathrm{c}}$, Alexander G. Hayes ${ }^{\mathrm{d}}$, \\ Patricio M. Rojo ${ }^{\mathrm{e}}$, Paul Corlies ${ }^{\mathrm{d}}$, Jason W. Barnes ${ }^{\mathrm{f}}$, Robert H. Brown ${ }^{\mathrm{g}}$, \\ Kevin H. Bainesh ${ }^{\mathrm{h}}$, Bonnie J. Buratti ${ }^{\mathrm{i}}$, Roger N. Clark ${ }^{\mathrm{j}}$, \\ Philip D. Nicholson ${ }^{\mathrm{k}}$, Christophe Sotin ${ }^{\mathrm{i}}$ \\ ${ }^{a}$ Astronomy Department, University of California, Berkeley, CA 94720-3411, USA \\ ${ }^{b}$ Department of Earth 83 Space Sciences, \\ University of California Los Angeles, Los Angeles, CA 90095, USA \\ ${ }^{c}$ Department of Atmospheric 83 Oceanic Sciences, \\ University of California Los Angeles, Los Angeles, CA 90095, USA \\ ${ }^{d}$ Center for Radiophysics and Space Research, \\ Cornell University, Ithaca, NY 14853, USA \\ ${ }^{e}$ Universidad de Chile, Camino El Observatorio 1515, Las Condes, Casilla 36-D \\ Santiago, Chile \\ ${ }^{f}$ Department of Physics, University of Idaho, Moscow, ID 83844-0903, USA \\ ${ }^{g}$ Lunar and Planetary Laboratory, University of Arizona, Tucson, AZ 85721, USA. \\ ${ }^{h}$ Space Science and Engineering Center, \\ University of Wisconsin, Madison, WI 53706, USA. \\ ${ }^{i}$ Jet Propulsion Laboratory, Caltech, Pasadena, CA, 91109 USA \\ ${ }^{j}$ United States Geological Survey, Denver, CO, 80225 USA \\ ${ }^{k}$ Department of Astronomy, Cornell University, Ithaca, NY 14853 USA
}

\section{Abstract}

The spatial distribution of the tropospheric methane on Titan was measured using near-infrared spectroscopy. Ground-based observations at $1.5 \mu \mathrm{m}$ (H-band) were performed during the same night using instruments with adaptive optics at both the W. M. Keck Observatory and at the Paranal Observatory on 17 July 2014 UT. The integral field observations with SIN-

Email address: mate@berkeley.edu (Máté Ádámkovics)

URL: http://astro.berkeley.edu/ madamkov (Máté Ádámkovics) 
FONI on the VLT covered the entire H-band at moderate resolving power, $R=\lambda / \Delta \lambda \approx 1,500$, while the Keck observations were performed with NIRSPAO near $1.5525 \mu \mathrm{m}$ at higher resolution, $R \approx 25,000$. The moderate resolution observations are used for flux calibration and for the determination of model parameters that can be degenerate in the interpretation of high resolution spectra. Line-by-line calculations of $\mathrm{CH}_{4}$ and $\mathrm{CH}_{3} \mathrm{D}$ correlated $k$ distributions from the HITRAN 2012 database were used, which incorporate revised line assignments near $1.5 \mu \mathrm{m}$. We fit the surface albedo and aerosol distributions in the VLT SINFONI observations that cover the entire H-band window and used these quantities to constrain the models of the highresolution Keck NIRSPAO spectra when retrieving the methane abundances. Cassini VIMS images of the polar regions, acquired on 20 July 2014 UT, are used to validate the assumption that the opacity of tropospheric aerosol is relatively uniform below $10 \mathrm{~km}$. We retrieved methane abundances at latitudes between $42^{\circ} \mathrm{S}$ and $80^{\circ} \mathrm{N}$. The tropospheric methane in the Southern mid-latitudes was enhanced by a factor of $\sim 10-40 \%$ over the nominal profile that was measured using the GCMS on Huygens. The Northern hemisphere had $\sim 90 \%$ of the nominal methane abundance up to polar latitudes $\left(80^{\circ} \mathrm{N}\right)$. These measurements suggest that a source of saturated polar air is equilibrating with dryer conditions at lower latitudes.

Keywords: Titan, atmosphere, Adaptive optics, Atmospheres, evolution, Atmospheres, structure 


\section{Introduction}

Methane $\left(\mathrm{CH}_{4}\right)$ is the most abundant condensible species on Titan, dominates the energy transport through the atmosphere (Mitchell, 2012), and is part of a complex hydrological cycle (Atreya et al., 2006; Roe, 2012). Clouds of methane can indicate regions of convection (e.g., Griffith et al., 2005), polar subsidence (Anderson et al., 2014), or evaporation from lakes (e.g., Brown et al., 2009; Turtle et al., 2009), while the formation of large scale methane cloud systems are diagnostic of atmospheric dynamics via their morphology (Mitchell et al., 2011) and how they evolve with time (Ádámkovics et al., 2010; Turtle et al., 2011a). The amount of methane near the surface is an important factor in triggering convective cloud formation (Barth and Rafkin, 2007) and in determining the strength of storms (Hueso and Sánchez-Lavega, 2006). Precipitation can return methane to the surface (Turtle et al., 2009, 2011a) where fluid transport has some role in closing the hydrological cycle. Seasonal variations in the general circulation (Mitchell et al., 2009) as well as predictions of the locations and frequency of clouds (Schneider et al., 2012) depend on the distribution of methane near the surface, both in the regolith and the lower atmosphere.

Lakes and seas of liquid hydrocarbons (Stofan et al., 2007) provide both sinks and sources of methane on the surface. The north pole contains by far the greatest extent of open liquids on Titan (Hayes et al., 2008; Lorenz et al., 2008; Sotin et al., 2012; Lorenz et al., 2014). The largest sea, Kraken Mare, extends down to $55^{\circ} \mathrm{N}$ at its southernmost point. A few low-latitude lake candidates have been suggested, one near the equator (Griffith et al., $2012 \mathrm{~b}$ ), and one at $40^{\circ} \mathrm{S}$ latitude (Vixie et al., 2014). The sole large lake in 
Titan's south polar region is Ontario Lacus (Turtle et al., 2009), although there are several basins that have been identified as potential "paleo-seas" that encompass a similar areal fraction to the northern seas (Hayes et al., 2011a).

Liquids presumably concentrate at the poles because they are the coldest points on the surface and therefore cold-traps for volatiles. However, the polar clustering might also be related to higher precipitation at the poles (Rodriguez et al., 2009; Brown et al., 2010; Rodriguez et al., 2011) relative to the dune-filled equatorial desert (Lorenz et al., 2006; Radebaugh et al., 2008; Le Gall et al., 2011; Rodriguez et al., 2014), which may be caused by circulation (Rannou et al., 2006; Friedson et al., 2009). The lower elevations of the poles relative to equatorial regions may also play a role (Iess et al., 2010; Lorenz et al., 2013). The reason for the pronounced North-South asymmetry in lake coverage is unknown. Aharonson et al. (2009) cited Milankovic-like cycles in Titan's orbital parameters as a possible explanation, which is supported by simulations of Titan's paleoclimate (Lora et al., 2014).

The physical properties of lakes are complicated by the fact that they are likely mixtures of hydrocarbons. Though methane composes Titan's raindrops, the seas may build up significant fractions of less-volatile ethane. Spectroscopic observations of Ontario Lacus suggest the presence of ethane (Brown et al., 2008), although the abundance is not constrained by these measurements. Recent observations of Ligeia Mare, conducted by the Cassini RADAR instrument, have demonstrated that it is primarily composed of methane (Mastrogiuseppe et al., 2014). Evaporation rates from a lake that is mostly methane will be much greater than from a lake that is mostly ethane 
(Mitri et al., 2007; Tokano, 2009). Lorenz (2014) points out that the ratio of methane to ethane may vary across lakes due to the concentration of solutes by heterogeneous evaporation and dilution by heterogeneous rainfall.

While shoreline recession at Ontario Lacus was reported over the timescale of the Cassini mission (Turtle et al., 2011b; Hayes et al., 2011b,a), the shoreline detection algorithms have been disputed (Cornet et al., 2012), leaving the contemporary evaporation rate over lakes uncertain. Cassini has observed albedo variations with both the Imaging Science Subsystem (ISS) and RADAR that depict smaller southern lakes disappearing between adjacent observations (Turtle et al., 2009; Hayes et al., 2011a), which was attributed to either infiltration or evaporation, although the rates could not be quantified. Changes in lake and sea volumes over geologic timescales have also likely occurred, as evidenced by the geomorphology of empty lakebeds in some polar terrains and the presence of drowned river valleys in the northern seas, which indicate that the liquid level is rising faster than fluvial sediment is being deposited (Hayes et al., 2008). Some of the lakebeds show a bright reflection near $5 \mu \mathrm{m}$, which is interpreted as a compositional signature that is attributed to the formation of organic evaporite (Barnes et al., 2011). The largest outcrop of evaporites are in the tropics, implying that these areas may have been seas during the geological past, perhaps under a different climatic regime (Moore and Howard, 2010; MacKenzie et al., 2014).

The evaporation of methane from surface lakes may have an observational impact on the atmosphere. Tokano (2014) recently revisited the Cassini radio occultation data (recorded from 2005-2009) and points out that retrievals assuming a uniform tropospheric methane distribution lead to surface pressure 
distributions that are inconsistent with the predictions of circulation models. Instead, Tokano (2014) argues for a substantially higher methane abundance in the Summer hemisphere. Penteado and Griffith (2010) searched for spatial variation in the methane abundance with high resolution Keck observations. The unsaturated lines of the resolved $3 \nu_{2}$ band of $\mathrm{CH}_{3} \mathrm{D}$ are sensitive to possible changes in the tropospheric methane abundance. Their measurements from December 2006 indicated that methane below $10 \mathrm{~km}$ altitude is constant to within $20 \%$ in the tropical atmosphere, sampled between the range of $32^{\circ} \mathrm{S}-18^{\circ} \mathrm{N}$. High resolution analysis with new methane lines lists (de Bergh et al., 2012) illustrates that significant improvements can be made in spectral fitting with recent laboratory data.

Here we present ground-based observations of the methane distribution on Titan using a methodology that improve upon the observing protocol and integration times of Penteado and Griffith (2010), and which are supported by both integral field observations from the same night, as well as a Cassini flyby from four days later. Our radiative transfer models include revised methane line lists from the most recent HITRAN database. The observations, data reduction, and calibrations are described in Section 2, while the radiative transfer model is detailed in Section 3. Results are presented in Section 4 and discussed in Section 5.

\section{Observations}

Observations were performed on 17 July 2014 UT at both the Paranal Observatory and the W. M. Keck Observatory. Instrumentation with complementary observing modes, resolutions, and bandpasses provided flux cal- 
ibration and characterization of the physical properties of the atmosphere and surface. Figure 1 illustrates the viewing geometry of the observations that are described below.

\subsection{VLT Observations}

The Spectrograph for INtegral Field Observations in the Near Infrared (SINFONI) on the Very Large Telescope (VLT) at Paranal Observatory was used as part of a campaign to monitor clouds on Titan. The spectrometer is fed by an adaptive optics module and uses two sets of stacked mirrors to optically divide and rearrange the field of view (FOV) into a single synthetic long slit that is spectrally dispersed by a grating onto the detector. We used the 0.8 " $\times 0.8$ " FOV, corresponding to a spatial pixel scale of $0.0125 " \times 0.0250 "$, with the grating that covers $1.45-2.45 \mu \mathrm{m}$ at a spectral resolution of $\Delta \lambda \approx 1 \mathrm{~nm}$, corresponding to a resolving power, $R=\lambda / \Delta \lambda \approx 1500$ (Eisenhauer et al., 2003). Four overlapping exposures with $2 \times 15$ sec coadds, offset by \pm 0.1 " from the disk center in both the $\mathrm{X}$ and $\mathrm{Y}$ directions of the FOV, are mosaicked to cover the entire disk.

Observations were reduced using version 2.5.2 of the SINFONI pipeline. The standard processing of the raw exposures includes correction of bad pixels, flat fielding, and correction of geometric distortions. The pipeline performs wavelength calibration and then reconstructs the 32 slices of spectra into a data cube. Sky frames are used to correct for sky emission.

The B3V type star Hip 74680 (HD 134485) was observed at an airmass of 1.019 and is used for both telluric correction and flux calibration. A high resolution H-band telluric template from ESO is convolved to the instrument resolution and scaled to fit telluric absorption features observed in the cal- 
ibration star near 1.47 and $1.58 \mu \mathrm{m}$; the normalized telluric template was scaled by a factor of 0.7 (and offset by 0.3 ), and then used to correct the target spectra.

Photometric calibration was performed by integrating the calibration star spectrum over the 2MASS filter curves in H-band and comparing with the known apparent magnitude. The relative spectral response $R_{\mathrm{H}}(\lambda)$, was used to determine the observed isoflux,

$$
I_{\mathrm{H}}=\frac{\int R_{\mathrm{H}}(\lambda) I_{\mathrm{obs}}(\lambda) d \lambda}{\int R_{\mathrm{H}}(\lambda) d \lambda},
$$

where $I_{\mathrm{obs}}(\lambda)$ is the observed spectrum. The 2MASS "zero-magnitude" reference flux in H-band from Cohen et al. (2003) is

$$
I_{\text {ref }}=1.133 \times 10^{-13} \pm 2.212 \times 10^{-15} \mathrm{~W} \mathrm{~cm}^{-2} \mu \mathrm{m}^{-1} .
$$

The spectral bandpass of SINFONI covers all of H-band, including the entire 2MASS H-band filter range, which facilitates the calibration of the NIRSPAO observations that cover a narrow band-pass at higher resolving power.

The apparent $\mathrm{H}$-band magnitude of the calibrator is $m_{\mathrm{H}}=8.322$ from 2MASS (Cutri et al., 2003), and the correction factor for converting the observed photon count rate $(\mathrm{DN} / \mathrm{s})$ to flux is given by

$$
c_{\mathrm{H}}=\frac{I_{\mathrm{ref}}}{I_{\mathrm{H}}} 10^{-m_{\mathrm{H}} / 2.5}
$$

The photometrically calibrated spectra, $I(\lambda)=I_{\mathrm{obs}}(\lambda) c_{\mathrm{H}}$, were then converted to units of reflectivity,

$$
\frac{I}{F}=\frac{r^{2}}{\Omega} \frac{I(\lambda)}{F_{\odot}(\lambda)},
$$


where $r$ is the heliocentric distance to Titan, $\Omega$ is the solid angle (in steradians) of each spatial pixel, and $\pi F_{\odot}(\lambda)$ is the solar spectrum at $1 \mathrm{AU}$, for which the 2000 ASTM Standard Extraterrestrial Spectrum Reference ${ }^{1}$ was used.

\subsection{Keck Observations}

The Near-InfraRed SPECctrometer (NIRSPEC) on the Keck II telescope at W. M. Keck Observatory (McLean et al., 1998) was used with the adaptive optics (AO) system, NIRSPEC+AO (NIRSPAO), for high resolution spectroscopy of Titan with a pixel scale of 0.018 " / pixel along the slit. The instrument was setup in the cross-dispersed echelle mode in $\mathrm{H}$-band with a $0.041 " \times 2.26$ " slit, giving a resolving power of $R \approx 25,000$. The echelle and cross-disperser angles were set to 62.8 and 36.5, respectively, nearly continuously covering the range $1.481-1.701 \mu \mathrm{m}$ in 7 echelle orders. The edges of neighboring orders can overlap with the 2.26 " slit in this setting. For this work we focused on Order 49, sampling the $1.541-1.563 \mu \mathrm{m}$ region, and where there was significant overlap at the edges of the order.

The standard ABBA dither (nod) pattern for the instrument moves the target $25 \%$ of the length of the slit $\left(0.57^{\prime \prime}\right)$ from center, which would position the limb of the $\sim 0.8$ " disk of Titan near the overlapping region of echelle order. This was avoided by using a smaller dither step along the slit. Sky exposures taken completely off target were recorded for sky subtraction. The integration time for each spectrum was $300 \mathrm{~s}$. Two ABBA sets were record with the slit aligned North-South near the central meridian, and one exposure

\footnotetext{
${ }^{1}$ http://rredc.nrel.gov/solar/spectra/am0/
} 
was taken with Titan in the center of the slit, for a total of 2,700 s integration on target, followed by a 300 s sky exposure. The spectral type A0V calibrator star was HD 141513 (Hip 77516). The logs for both SINFONI and NIRSPAO observations are presented in Table 1.

The NIRSPAO data are reduced using standard procedures for bad pixel correction, flat fielding, and cosmic ray rejection. The spatial and spectral rectification routines from the REDSPEC package are used before shifting and stacking exposures. A bare sky exposure is used for first order sky subtraction. A second order correction was performed by (1) offsetting each exposure to the median of the stacked set near the edge of the order, and then (2) scaling each exposure to the median value at the center of the order. The stack of images for individual exposures was collapsed using the mean of each pixel.

Flux calibration of an extended object in a slit spectrometer can be challenging because the slit losses due to the point spread function (PSF) extending over the edge of the slit need to be determined. For point sources, it can often be assumed that the unknown slit losses for the calibrator and the target are the same. However, this is not the case when comparing a point source calibrator and an extended target. After an initial telluric and flux calibration was performed using Hip 77516 (HD 141513), the NIRSPAO data were then scaled to match the calibrated SINFONI observations. Two wavelengths were considered that are sensitive to the surface and lower atmosphere, Figure 2.

Unlike the narrow NIRSPAO channels, which can probe regions of high methane opacities near $1.55 \mu \mathrm{m}$ and therefore the atmosphere, the SINFONI 
channels near $1.55 \mu \mathrm{m}$ are broad, cover regions of predominantly low methane opacity, and are therefore more sensitive to the surface. Due to the difference in bandpass, the reflectance along the NIRSPAO slit at $1.5555 \mu \mathrm{m}$ does not match the corresponding SINFONI channel and is compared to the $1.6145 \mu \mathrm{m}$ SINFONI channel in Figure 2. The $1.55 \mu \mathrm{m}$ spectral region is generally thought to be a surface-sensitive on Titan, however, when sampled at high spectral resolution there are wavelengths in this region with large gas opacity that probe only the atmosphere.

\subsection{Cassini VIMS Observations}

Spectral mapping cubes were obtained by Cassini VIMS (Brown et al., 2004) during both the ingress and egress of the T103 flyby on 2014 Jul 20 UT, and provide views of both polar regions. We reduce the VIMS IR channels from two cubes (datasets 1784502376_1 and 1784584782_1) using the standard pipeline for calibration and determination of the viewing geometry. Images from the $1.573 \mu \mathrm{m}$ and $1.625 \mu \mathrm{m}$ channels are used to evaluate meridional variation in near-surface hazes toward both poles. Since the tropospheric haze opacity near the surface can be degenerate with the methane abundance there (described below) we use the VIMS observations to differentiate between models.

\section{Radiative Transfer}

We implement a model of the atmosphere using the in situ measurements made with instruments on the Huygens probe, which provide structure, chemical composition, and aerosol scattering in our reference model. Methane line assignments from the HITRAN 2012 database (Rothman et al., 2013) 
are used to determine gas opacities via line-by-line methods and we solve the radiative transfer using the discrete ordinates method (Stamnes et al., 1988).

\subsection{Structure and Composition}

We use a model with 20 layers, which have boundaries (levels) that are evenly spaced in pressure above and below $300 \mathrm{mbar}(\mathrm{hPa})$, covering the 2.75 - 1466.45 mbar range sampled by the Huygens Atmospheric Structure Instrument (HASI) on Cassini (Fulchignoni et al., 2005). This corresponds to an altitude range from the surface up to $147 \mathrm{~km}$. There are 10 layers sampled at $\sim 30$ mbar intervals through the tropopause and stratosphere and 10 layers sampling the troposphere at $\sim 115$ mbar steps. Fewer layers can improve the computational speed, but yield inconsistent calculations, whereas increasing the number of layers above 20 has no significant benefit. Altitude sensitivity for cloud retrievals may be improved with additional layers, but this topic is beyond the scope of this work. After the levels in the model are determined, the pressures, temperatures and densities for each layer are interpolated and the total column densities are determined using the mole fractions reported by the Huygens Gas Chromatograph Mass Spectrometer (GCMS)

(Niemann et al., 2010). The atmospheric structure and composition are tabulated in Table 2 .

subsectionAerosol Model

The aerosol scattering phase functions and opacities were measured in situ by the the Descent Imager-Spectral Radiometer (DISR) on the Huygens probe (Tomasko et al., 2008). We fit 32nd order Legendre polynomials to the phase functions tabulated at $1.29 \mu \mathrm{m}, 1.58 \mu \mathrm{m}$, and $2.00 \mu \mathrm{m}$, using a Levenberg-Marquardt (LM) optimization, and linearly interpolate coeffi- 
cients for intermediate wavelengths. The vertical opacity structure from the model of (Tomasko et al., 2008) is used, with a haze single scattering albedo, $\omega_{H}=0.96$.

\section{2. $\mathrm{CH}_{4}$ and $\mathrm{CH}_{3} \mathrm{D}$ opacities}

Spectra resolving the natural line shape of $\mathrm{CH}_{4}$ and $\mathrm{CH}_{3} \mathrm{D}$ are calculated using line-by-line methods. These spectra are used to calculate correlated- $k$ coefficients at the resolution and dispersion plate scale of both the NIRSPAO and SINFONI instruments. Correlated- $k$ values (Lacis and Oinas, 1991) for $\mathrm{CH}_{4}$ and $\mathrm{CH}_{3} \mathrm{D}$ are calculated for 390 combinations of temperature and pressure that have been used in the literature, e.g., by Sromovsky et al. (2012) and Irwin et al. (2014). $\mathrm{CH}_{4}$ and $\mathrm{CH}_{3} \mathrm{D}$ lines are from the HITRAN 2012 database (Rothman et al., 2013), which include the WKMC-80K methane line data of Campargue et al. (2012) that are relevant to this spectral region.

We calculate the monochromatic opacity at frequency, $\nu$, pressure $P$, and temperature, $T$, by summing over lines, $\ell$, as described by Sromovsky et al. (2012),

$$
k(\nu ; P, T)=\sum_{\ell} S_{\ell}\left(T_{0}\right) \exp \left[\frac{h c E_{\ell}}{k_{B}}\left(\frac{1}{T_{0}}-\frac{1}{T}\right)\right] \frac{Q\left(T_{0}\right)}{Q(T)} f_{\ell}\left(\nu-\nu_{\ell} ; P, T\right),
$$

correcting the typo of the reversed $T_{0}$ and $T$ in the square brackets of their Equation 1. $S_{\ell}\left(T_{0}\right)$ is the line strength at reference temperature $T_{0}, E_{\ell}$ is the lower state energy of the line, and the partition function ratio $Q\left(T_{0}\right) / Q(T)$ is approximated by $\left(T_{0} / T\right)^{3 / 2}$. The speed of light, Planck and Boltzmann constants are $c, h$, and $k_{B}$, respectively.

The line shape function, $f_{\ell}$, is given by the Voigt profile with a correction 
to the Lorentz far wing, $\chi\left(\nu-\nu_{0}\right)$.

$$
f_{\ell}\left(\nu-\nu_{0} ; P, T\right)=V\left(\nu_{0} ; \alpha_{D}, \alpha_{L}\right) \chi\left(\nu-\nu_{0}\right)
$$

where

$$
\int_{-\infty}^{\infty} f_{\ell}(\nu) d \nu=1
$$

Various prescriptions for $\chi$ are in the literature and we use the sub-Lorenztian line shape of Campargue et al. (2012) that is determined from laboratory data. The following notation is used to describe the Voigt profile when determining line shape:

$$
\mathcal{V}(x ; \sigma, \gamma)=\int_{-\infty}^{\infty} G\left(x^{\prime} ; \sigma\right) L\left(x-x^{\prime} ; \gamma\right) d x^{\prime}
$$

where

$$
G(x ; \sigma) \equiv \frac{e^{-x^{2} /\left(2 \sigma^{2}\right)}}{\sigma \sqrt{2 \pi}} \quad \text { and } \quad L(x ; \gamma) \equiv \frac{\gamma}{\pi\left(x^{2}+\gamma^{2}\right)}
$$

The integral in Equation 7 can be evaluated with the real part of the complex error function. The Voigt line shape in terms of physical parameters is given by:

$$
V\left(\nu_{0} ; \alpha_{D}, \alpha_{L}\right)=\mathcal{V}(\sigma, \gamma) \sqrt{\frac{\ln (2)}{\pi}} \alpha_{D}
$$

where the dimensionless parameters

$$
\sigma=\frac{\nu-\nu_{0}}{\alpha_{D}} \sqrt{\ln (2)} \text { and } \gamma=\frac{\alpha_{L}}{\alpha_{D}} \sqrt{\ln (2)}
$$

are given in terms of the following physical parameters

$$
\alpha_{D}=\frac{\nu_{0}}{c} \sqrt{\frac{2 k T}{m}} \text { and } \alpha_{L}=\gamma_{a i r} \frac{P}{P_{0}}\left(\frac{T_{0}}{T}\right)^{n}
$$

respectively, using the transition frequency, $\nu_{0}$, the molecular mass, $m$, temperature, $T$, together with the reference line broadening half-width, $\gamma_{\text {air }}$ that is determined at a standard pressure $P_{0}$ and temperature $T_{0}$, and varies with some temperature dependent exponent, $n$. 


\subsection{Practical Implementation}

The code described for setting up the atmospheric opacity structure and solving the radiative transfer is original to this work and implemented as a Python package and that is publicly available ${ }^{2}$, including a Python implementation of $\mathrm{CDISORT}^{3}$ (PyDISORT). The Python package management tools should facilitate installing and compiling the code. Reference data files can be downloaded using methods within the atmosphere package.

\section{Results}

The reference atmospheric model described in Section 3 is based on the DISR, HASI, and GCMS measurements. It is most applicable to the tropical regions of Titan during the epoch of the Huygens probe descent in 2005. The distribution of aerosol in the atmosphere, which is critical for the calculation of near-IR spectra, is known to vary on seasonal timescales (e.g., Lorenz et al., 2004), however, there is no predictive model for the global vertical structure of aerosol during the epoch of the observations studied here, so we fit the SINFONI observations to retrieve the aerosol structure (e.g., Ádámkovics et al., 2006).

Synthetic spectra calculated using models of the aerosol scattering and structure made using DISR (Tomasko et al., 2008) can overestimate the observed intensities by a factor of up to $\sim 2$. Reducing the aerosol single scattering albedo, $\omega_{H}$, reducing the aerosol opacity, or changing the scattering phase function can each reduce intensities in synthetic spectra. de Bergh

\footnotetext{
${ }^{2}$ https://github.com/adamkovics/atmosphere

${ }^{3}$ http://www.libradtran.org
} 
et al. (2012) and Griffith et al. (2012a) fit VIMS observations in the Hband window by decreasing $\omega_{H}$ to 0.94 and removing the back-scattering portion of the phase function below $80 \mathrm{~km}$. That is, they use the high altitude DISR phase function throughout the atmosphere. The overestimated intensities can also occur at longer wavelengths (e.g., $2 \mu \mathrm{m})$ where there is no back-scattering peak in the DISR aerosol model. The scattering albedo is unconstrained beyond the wavelength range of DISR observations, so $\omega_{H}$ can be decreased to reconcile synthetic spectra with observations (e.g., Griffith et al., 2012a; Hirtzig et al., 2013). There is significant degeneracy among the aerosol properties when interpreting spectra, and a critical evaluation of the aerosol scattering is beyond the scope of this work.

Here we assume that the aerosol single scattering albedo and phase functions, which are related to the composition and morphology of the particles, are the same as measured by DISR on the Huygens probe. We set $\omega_{H}=0.96$ and do not vary the phase function, and instead consider changes in the aerosol optical depth.

We use the vertical profile measured by DISR (Tomasko et al., 2008) as a guide and do not treat the aerosol opacity in each model layer as a free parameter. That is, we do not consider arbitrary aerosol vertical structures. Instead, we consider two cases for variation in the aerosol vertical structure: (1) We assume that the tropospheric aerosol is uniform below $10 \mathrm{~km}$ and that the aerosol opacity above this altitude can be scaled linearly from the DISR measurements by a factor, $f_{H}$. This approximation treats that spatial variation in haze with a single parameter. (2) In order to explore the decoupling between tropospheric and stratospheric aerosol, we consider two independent 
scale factors for the hazes above and below $65 \mathrm{~km}$. Neither of these models is motivated by microphysics, nor do they take into account empirical evidence for the variations in the aerosol scattering. However, they are sufficient for interpreting the observations.

Synthetic spectra are compared to observations using a standard quality metric,

$$
\hat{\chi}^{2}=\frac{1}{N} \sum_{i}\left(\frac{r_{i}}{\sigma_{\mathrm{obs}}}\right)^{2},
$$

where the residual between the observation and model, $r_{i}$, for each data point, $i$, is normalized by the observational uncertainty, $\sigma_{\mathrm{obs}}$, and summed over a total of $N$ data points. We conservatively estimate that $\sigma_{\mathrm{obs}}=0.005$ for the SINFONI observations. A $\hat{\chi}^{2} \lesssim 1$ generally suggests an acceptable model.

Synthetic spectra are generated for the various aerosol models discussed above and compared with SINFONI observations by calculating $\hat{\chi}^{2}$, listed in Table 3. Assuming that the DISR aerosol model for the aerosol scattering and vertical structure applies at all latitudes is inconsistent with the SINFONI spectra, with $\hat{\chi}^{2} \sim 5$. Removing the backscattering component in the aerosol scattering phase function, $P(\theta)$, leads to a better fit, but with $\hat{\chi}^{2} \gtrsim 3$. Changing both $P(\theta)$ and $\omega_{H}$ according to de Bergh et al. (2012) is a further improvement, but still not statistically consistent with the observations, since $\hat{\chi}^{2} \sim 3$. Although synthetic spectra are within $\sim 10 \%$ of the observations near the center of the disk, the intensities are over-estimated near to the limb. Using the DISR model for scattering and vertical structure, while scaling the entire column of aerosol by a factor $f_{H}=0.5$, and assuming this structure applies at all latitudes is a significant improvement 
with $\hat{\chi}^{2} \approx 1$. The decrease in aerosol column is degenerate with the scattering properties of the aerosol, such that including a spurious back-scattering peak at low altitudes would cause an over-estimate of the reduction in aerosol opacity.

The hazes on Titan are known to not be uniform, so we fit the SINFONI spectra with two variable haze models. We use a Levenberg-Marquardt (LM) optimization is to determine the surface albedo, $A_{S}$, and either one or two haze scale factors, $f_{H}$, for each of the SINFONI spectra, which we refer to as "2-parameter" and "3-parameter" models, respectively. The free parameters are well-constrained with reasonable initial estimates, justifying the LM optimization., SINFONI spectra along the region corresponding to the NIRSPAO slit are considered, and 12 of these spectra are plotted as examples in Figure 3, illustrating that these parameters are sufficient for interpreting the observations. With $\hat{\chi}^{2} \sim 0.7$ and 0.6 for the 2- and 3-parameter models, respectively, the variable haze models are significantly better at reproducing the observations than by assuming the same aerosol vertical profile at all latitudes. The measurements cover a range of viewing geometries from the center of the disk to the limb, with the limb being most sensitive to the scattering phase function. The 3-parameter model produces a better fit to the 1.65-1.70 $\mu \mathrm{m}$ region (sensitive to the stratosphere) at latitudes south of $20^{\circ} \mathrm{N}$, where a comparatively large decrease in tropospheric aerosol is compensated by a smaller decrease in stratospheric aerosol.

The optimized parameters for $A_{S}$ and $f_{H}$ from the SINFONI observations are plotted in Figure 4. These values are interpolated onto the NIRSPAO pixel scale and constrain the NIRSPAO retrievals. The hemispheric asym- 
metry in $A_{S}$ that is seen in the SINFONI observations and the reprojected VIMS map, Figure 1, is also observed in the NIRSPAO profile at $1.5581 \mu \mathrm{m}$. While it is useful to have a benchmark measurement of $A_{S}$, there are roughly $16 \mathrm{hrs}$ between the NIRSPAO and SINFONI observations, over which Titan rotates $\sim 16^{\circ}$, (Table 1). Due to the timespan between observations, we allow for an offset of the surface albedo, $\delta A_{S}$, to be a free parameter in fit of the NIRSPAO observations. The differences in surface albedo at the times of the two observations can be seen by comparing the red and black dashed lines in Figure 2 .

The interpolated values of $f_{H}$ are used to generate the atmospheric structure for fitting the NIRSPAO observations. Once again we use an LM optimization, in this case for three free parameters for spectra at each pixel along the slit: (1) an adjustment to the input surface albedo, $\delta A_{S},(2)$ a high altitude haze factor, $f_{H}^{\prime}$, which scales the opacity in the uppermost layer of the model and accounts for the fact that the 2-parameter model doesn't necessarily fit spectral regions sensitive to the stratosphere, such as the low reflectance region in the NIRSPAO observations, and (3) a scale factor for the tropospheric methane (both $\mathrm{CH}_{4}$ and $\mathrm{CH}_{3} \mathrm{D}$ ) abundances that were measured by the GCMS on Huygens, $f_{\mathrm{CH} 4}$.

The best fit spectra from 4 out of 42 pixels on the disk are presented in Figure 5, demonstrating the $\mathrm{S} / \mathrm{N}$ obtained in the observations for one pixel along the slit and the agreement between models and observations. Both models fit the observations equally well, with residuals that are generally smaller than the estimated observational uncertainty. The estimate of the pixel-to-pixel noise in the observations in determined by taking the stan- 
dard deviation of 5 pixels centered on the spectrum of interest. This is an overestimate of noise since the 5 pixels probe different spatial locations on the disk. This estimate also ignores systematic uncertainties beyond pixelto-pixel noise variations. The $1 \sigma$ uncertainties are illustrated as the shaded gray regions in the residuals panel for each spectrum in Figure 5. Parameters for each model are in the legend.

The LM optimization is performed for all spectra from $42^{\circ} \mathrm{S}$ to $80^{\circ} \mathrm{N}$ using both haze models, assuming either spatially uniform haze below $10 \mathrm{~km}$ or variable haze in this altitude region. Uncertainties are estimated using the roots of the diagonal elements of the $3 \times 3$ covariance matrix of the LM optimization. A plot of the latitudinal variation of $f_{\mathrm{CH} 4}$ is presented in Figure 6 for both model assumptions. In the case of uniform haze below $10 \mathrm{~km}$, there is a significant increase in tropospheric methane toward the Southern Hemisphere and a slight depletion in the Northern temperate regions, to $\sim 90 \%$ of the value measured by the GCMS on Huygens. If the tropospheric aerosol is assumed to be variable, then the methane abundance is essentially uniform. The degeneracy between the tropospheric haze and methane is due to the broadening and blending of methane lines at pressures above a bar. Increasing methane near the surface leads to smaller reflectivity at surfaceprobing wavelengths in a manner similar to decreasing tropospheric aerosol. Independently constraining the spatial variation in tropospheric aerosol can break the degeneracy between these two models.

Three days after the ground-based observations there was a Cassini flyby (T103) that passed over the Southern Hemisphere on ingress and over the Northern Hemisphere on egress, with VIMS recording global views of the po- 
lar regions of Titan. Figures 7 show VIMS images from the $1.5 \mu \mathrm{m}$ window in channels that are sensitive to the surface $(1.573 \mu \mathrm{m})$ and a neighboring channel $(1.625 \mu \mathrm{m})$ where the contribution from surface is small. Differences in reflected intensity due to surface albedo variation can dominate those due to spatial variation in haze, so we calculate synthetic images for both channels, considering either uniform haze, or a haze with a gradient of increasing opacity toward the North.

The synthetic $1.573 \mu \mathrm{m}$ images in Figure 7 have decreasing surface albedo contrast toward the limb and the terminator, due to the increasing path length through the atmosphere associated with large incidence or emission angles. The greatest albedo contrast is near the dark, polar lakes in the Northern Hemisphere and near mid-latitudes in the Southern Hemisphere. Assuming a haze gradient leads to a brighter region near the pole, and slightly greater surface albedo contrast near the South Pole. In general, there are only minor differences in the synthetic images of the surface when comparing the two haze models.

The synthetic $1.625 \mu \mathrm{m}$ images, however, illustrate an increased sensitivity to the aerosol model. A uniform haze leads to limb brightening that is consistent with observations both in the North and the South polar regions, whereas the gradient in the haze leads to brighter Northern, and a darker Southern, polar regions than are observed by VIMS. A uniform distribution of aerosol near the surface means that the NIRSPAO spectra and are more consistent with a meridional variation in methane, and not a variation in aerosol. 


\section{Discussion}

We have used complementary ground-based observations from NIRSPAO at Keck and SINFONI at the VLT to measure the tropospheric methane distribution on Titan. The spatially-resolved SINFONI observations at moderate resolution, across the entire H-band, are used for the flux calibration and constraining the aerosol haze distribution, while the high resolution NIRSPAO observations are sensitive to the tropospheric methane. We used HITRAN 2012 line lists to generate $k$ distributions for $\mathrm{CH}_{4}$ and $\mathrm{CH}_{3} \mathrm{D}$ at the resolutions and plate scales that correspond to each of the observations. Levenberg-Marquardt optimization was used to fit the observations, and retrieve the tropospheric methane abundance, assuming either variable or uniform tropospheric haze. Cassini VIMS images suggest that the haze is uniform toward both poles and that there is meridional variation in methane.

The distribution of methane in Figure 6 reveals two interesting features. First, it is nearly uniform in the northern hemisphere from $15^{\circ} \mathrm{N}$ to the pole, and marginally lower $(\sim 10 \%)$ than the Huygens GCMS measurement. Second, concentrations rise monotonically to the south of $15^{\circ} \mathrm{N}$ and reach a peak value of 1.2-1.6 times the Huygens GCMS measurement at $40^{\circ} \mathrm{S}$. This suggests that there are at least two, distinct source regions of methane vapor, and that the atmosphere is mixing the air masses from these source regions in latitudes south of $15^{\circ} \mathrm{N}$. If we assume the source regions produce saturated air masses at the local temperature, we can estimate the difference in their temperatures. For a given temperature difference, $\Delta T$, the ClausiusClapeyron relation predicts a fractional change in saturation vapor pressure, $\Delta e_{s} / e_{s} \approx L_{v} \Delta T /\left(R_{v} T^{2}\right)$, were $L_{v}$ is the latent heat of vaporization and $R_{v}$ is 
the methane gas constant. Assuming the northern hemisphere source region has a temperature of $\sim 91 \mathrm{~K}$, a $40 \%$ change in the saturation vapor pressure requires the source regions have a temperature difference of $\sim 3 \mathrm{~K}$, which is approximately the largest observed surface brightness temperature difference (Jennings et al., 2011).

Evaporation from Ontario Lacus (Turtle et al., 2011b; Hayes et al., 2011b) as the sole explanation for methane enhancement (during southern summer) was rejected by Tokano (2014) based on the small spatial coverage of Ontario Lacus (0.04\% of the Southern hemisphere) and the assumption of $\sim 4 \mathrm{~m}$ change in lake depth. This suggests that lake evaporation at the poles over the timescale of the Cassini mission is an unlikely explanation for the increase in methane from the equator toward southern mid-latitudes at the onset of southern winter. However, evaporation from moist ground or a number of small, methane-dominated lakes is still possible. Indeed, evaporation rates from moist surfaces can exceed the rates from standing liquids due to the larger surface area available and the possibility for increased turbulence above a rough surface. Our results suggest that evaporation from the surface, in a region poleward of a particular latitude, is increasing the relative humidity of the atmosphere. The observed gradient in methane abundance (toward the winter pole) may then be an indication of a relatively moist air parcel from the pole equilibrating to the drier conditions at the equator during meridional transport.

In November 2000, Anderson et al. (2008) used spectral images from 0.6 - $1.0 \mu \mathrm{m}$, obtained with the Hubble Space Telescope (HST), to measure a latitudinal gradient from the south pole toward the northern (winter) mid- 
latitudes. They report that the tropospheric methane column roughly doubled from $\sim 70^{\circ} \mathrm{S}$ to $\sim 10^{\circ} \mathrm{N}$, and were presumably sensitive to to the near surface humidity. Since the HST observations were nearly a half Titan-year earlier, the seasons should be analogous to our ground-based observations, but mirrored North to South. One difference between the measurements is that Anderson et al. (2008) report a significant drop in the methane column in their northern-most datapoint at $\sim 30^{\circ} \mathrm{N}$, whereas our measurements increase through $\sim 40^{\circ} \mathrm{S}$. Another consideration is that Anderson et al. (2008) report a much weaker, or absent, latitudinal variation in methane 7 days earlier at a different central meridian longitude (CML), which they speculate is an indication of either a surface or sub-surface source of methane that is spatially variable.

An increase in methane toward the winter polar is at odds with the predictions of circulation models that have been used to interpret Cassini radio occultation data. The measurements obtained over the period from southern summer to southern equinox (2006-2009) indicate that tropospheric methane should increase from the equator toward the Summer pole (Tokano, 2014). The latitudinal gradient in sea-level pressures that are retrieved by Tokano (2014), assuming that methane increases toward the Summer pole, are consistent with the location of the seasonal convergence zone and the observed regions of precipitation as tracked by cloud formation (Mitchell, 2012). Further work is required to determine conclusively if these observations test particular assumptions of circulation models, e.g., the distribution of methane at the surface, or if some other mechanism needs to be invoked to interpret the measurements. For example, episodic releases of methane, from some 
form of cryovolcanism, have been suggested as mechanism for supplying the moisture to southern mid-latitude clouds (Roe et al., 2005). However, the rate of cloud occurrence in the South has dropped with the changing seasons and the locations of clouds are generally thought to be controlled by circulation, rather than topography (Roe, 2012; Mitchell, 2012). Nonetheless, the properties of the surface regolith remain a mystery and the potential for the episodic release of methane near polar latitudes is unconstrained.

Repeated ground-based observations in different epochs, and at different CML can test for contemporary variability is near-surface sources of methane. If mixing of saturated polar air during transport controls the gradient in tropospheric methane, we can predict that the gradient in methane should change seasonally with the changing circulation. Changes in the gradient on more rapid time-scales, or at different CML can confirm either episodic releases of methane or spatial variation in the source region. Simultaneous observations with IFU and slit spectrometers, and quantitative constraints on the surface albedo from VIMS maps could further constrain our models. Degeneracies in the parameterized properties of the haze could also be further constrained by aerosol microphysical models that predict how the vertical structure of aerosol opacity and scattering phase functions change with time. Continuing work on methane line assignments, line shapes, and possible variations in upper stratospheric methane (e.g., Lellouch et al., 2014) can be used to make quantitative improvements to both the relative and absolute uncertainties in the retrieval.

We have presented measurements of the meridional variation in tropospheric methane on Titan. These results suggest that localized regions of 
evaporation occur at Southern polar latitudes in the winter, likely from a moist regolith. The simultaneous analysis of Keck NIRSPAO, VLT SINFONI, and Cassini VIMS observations illustrate the challenges in performing an accurate retrieval. We have mentioned a few improvements for constraining assumptions in our radiative transfer models and have suggested additional observations of this type at future epochs. Accurately measuring the spatial variation in the tropospheric methane will constrain sources of methane at the surface and inform our understanding of the hydrological cycle on Titan.

\section{Acknowledgements}

This work was supported by NASA PAAST grants NNX14AG82G and NNX12AM81G. MÁ was supported in part by NSF AST-1008788. PMR was supported by Fondecyt grant \#1120299. We wish to acknowledge Jonathan I. Lunine, Elizabeth P. Turtle, and Valentin D. Ivanov, who are members of the VLT SINFONI cloud observing campaign that provided the SINFONI observations presented here. Some of the data presented were obtained at the W. M. Keck Observatory, which is operated as a scientific partnership among the California Institute of Technology, the University of California and the National Aeronautics and Space Administration. The Observatory was made

possible by the generous financial support of the W. M. Keck Foundation. The authors wish to recognize the significant cultural role that the summit of Mauna Kea has always had within the indigenous Hawaiian community. We are fortunate to have the opportunity to conduct observations from this mountain. 


\section{References}

Ádámkovics, M., Barnes, J. W., Hartung, M., de Pater, I., Aug. 2010. Observations of a stationary mid-latitude cloud system on Titan. Icarus 208, 868-877.

Ádámkovics, M., de Pater, I., Hartung, M., Eisenhauer, F., Genzel, R., Griffith, C. A., Jun. 2006. Titan's bright spots: Multiband spectroscopic measurement of surface diversity and hazes. Journal of Geophysical Research (Planets) 111 (E7), E07S06.

Aharonson, O., Hayes, A. G., Lunine, J. I., Lorenz, R. D., Allison, M. D., Elachi, C., Dec. 2009. An asymmetric distribution of lakes on Titan as a possible consequence of orbital forcing. Nature Geoscience 2, 851-854.

Anderson, C. M., Samuelson, R. E., Achterberg, R. K., Barnes, J. W., Flasar, F. M., Nov. 2014. Subsidence-induced methane clouds in Titan's winter polar stratosphere and upper troposphere. Icarus 243, 129-138.

Anderson, C. M., Young, E. F., Chanover, N. J., McKay, C. P., Apr. 2008. HST spectral imaging of Titan's haze and methane profile between 0.6 and $1 \mu \mathrm{m}$ during the 2000 opposition. Icarus 194, 721-745.

Atreya, S. K., Adams, E. Y., Niemann, H. B., Demick-Montelara, J. E., Owen, T. C., Fulchignoni, M., Ferri, F., Wilson, E. H., Oct. 2006. Titan's methane cycle. Planet. Space Sci. 54, 1177-1187.

Barnes, J. W., Bow, J., Schwartz, J., Brown, R. H., Soderblom, J. M., Hayes, A. G., Vixie, G., Le Mouélic, S., Rodriguez, S., Sotin, C., Jaumann, R., 
Stephan, K., Soderblom, L. A., Clark, R. N., Buratti, B. J., Baines, K. H., Nicholson, P. D., Nov. 2011. Organic sedimentary deposits in Titan's dry lakebeds: Probable evaporite. Icarus 216, 136-140.

Barth, E. L., Rafkin, S. C. R., Feb. 2007. TRAMS: A new dynamic cloud model for Titan's methane clouds. Geophys. Res. Lett. 34 (3), L03203.

Brown, M. E., Roberts, J. E., Schaller, E. L., Feb. 2010. Clouds on Titan during the Cassini prime mission: A complete analysis of the VIMS data. Icarus 205, 571-580.

Brown, M. E., Smith, A. L., Chen, C., Ádámkovics, M., Nov. 2009. Discovery of Fog at the South Pole of Titan. Astrophys. J. Lett. 706 (1), L110-L113.

Brown, R. H., Baines, K. H., Bellucci, G., Bibring, J.-P., Buratti, B. J., Capaccioni, F., Cerroni, P., Clark, R. N., Coradini, A., Cruikshank, D. P., Drossart, P., Formisano, V., Jaumann, R., Langevin, Y., Matson, D. L., McCord, T. B., Mennella, V., Miller, E., Nelson, R. M., Nicholson, P. D., Sicardy, B., Sotin, C., Dec. 2004. The Cassini Visual And Infrared Mapping Spectrometer (Vims) Investigation. Space Science Reviews 115, 111-168.

Brown, R. H., Soderblom, L. A., Soderblom, J. M., Clark, R. N., Jaumann, R., Barnes, J. W., Sotin, C., Buratti, B., Baines, K. H., Nicholson, P. D., Jul. 2008. The identification of liquid ethane in Titan's Ontario Lacus. Nature 454, 607-610.

Campargue, A., Wang, L., Mondelain, D., Kassi, S., Bézard, B., Lellouch, E., Coustenis, A., Bergh, C. d., Hirtzig, M., Drossart, P., May 2012. An 
empirical line list for methane in the $1.26-1.71 \mu \mathrm{m}$ region for planetary investigations ( $\mathrm{T}=80-300 \mathrm{~K})$. Application to Titan. Icarus 219, 110-128.

Cohen, M., Wheaton, W. A., Megeath, S. T., Aug. 2003. Spectral Irradiance Calibration in the Infrared. XIV. The Absolute Calibration of 2MASS. AJ 126, 1090-1096.

Cornet, T., Bourgeois, O., Le Mouélic, S., Rodriguez, S., Sotin, C., Barnes, J. W., Brown, R. H., Baines, K. H., Buratti, B. J., Clark, R. N., Nicholson, P. D., Jul. 2012. Edge detection applied to Cassini images reveals no measurable displacement of Ontario Lacus' margin between 2005 and 2010. Journal of Geophysical Research (Planets) 117, 7005.

Cutri, R. M., Skrutskie, M. F., van Dyk, S., Beichman, C. A., Carpenter, J. M., Chester, T., Cambresy, L., Evans, T., Fowler, J., Gizis, J., Howard, E., Huchra, J., Jarrett, T., Kopan, E. L., Kirkpatrick, J. D., Light, R. M., Marsh, K. A., McCallon, H., Schneider, S., Stiening, R., Sykes, M., Weinberg, M., Wheaton, W. A., Wheelock, S., Zacarias, N., Mar. 2003. 2MASS All-Sky Catalog of Point Sources (Cutri+ 2003). VizieR Online Data Cat$\operatorname{alog} 2246,0$.

de Bergh, C., Courtin, R., Bézard, B., Coustenis, A., Lellouch, E., Hirtzig, M., Rannou, P., Drossart, P., Campargue, A., Kassi, S., Wang, L., Boudon, V., Nikitin, A., Tyuterev, V., Feb. 2012. Applications of a new set of methane line parameters to the modeling of Titan's spectrum in the 1.58 $\mu \mathrm{m}$ window. Planet. Space Sci. 61, 85-98. 
Eisenhauer, F., Abuter, R., Bickert, K., Biancat-Marchet, F., Bonnet, H., Brynnel, J., Conzelmann, R. D., Delabre, B., Donaldson, R., Farinato, J., Fedrigo, E., Genzel, R., Hubin, N. N., Iserlohe, C., Kasper, M. E., Kissler-Patig, M., Monnet, G. J., Roehrle, C., Schreiber, J., Stroebele, S., Tecza, M., Thatte, N. A., Weisz, H., Mar. 2003. SINFONI - Integral field spectroscopy at 50 milli-arcsecond resolution with the ESO VLT. In: Instrument Design and Performance for Optical/Infrared Ground-based Telescopes. Edited by Iye, Masanori; Moorwood, Alan F. M. Proceedings of the SPIE, Volume 4841, pp. 1548-1561 (2003). pp. 1548-1561.

Friedson, A. J., West, R. A., Wilson, E. H., Oyafuso, F., Orton, G. S., Dec. 2009. A global climate model of Titan's atmosphere and surface. Planet. Space Sci. 57, 1931-1949.

Fulchignoni, M., Ferri, F., Angrilli, F., Ball, A. J., Bar-Nun, A., Barucci, M. A., Bettanini, C., Bianchini, G., Borucki, W., Colombatti, G., Coradini, M., Coustenis, A., Debei, S., Falkner, P., Fanti, G., Flamini, E., Gaborit, V., Grard, R., Hamelin, M., Harri, A. M., Hathi, B., Jernej, I., Leese, M. R., Lehto, A., Lion Stoppato, P. F., López-Moreno, J. J., Mäkinen, T., McDonnell, J. A. M., McKay, C. P., Molina-Cuberos, G., Neubauer, F. M., Pirronello, V., Rodrigo, R., Saggin, B., Schwingenschuh, K., Seiff, A., Simões, F., Svedhem, H., Tokano, T., Towner, M. C., Trautner, R., Withers, P., Zarnecki, J. C., Dec. 2005. In situ measurements of the physical characteristics of Titan's environment. Nature 438, 785-791.

Griffith, C. A., Doose, L., Tomasko, M. G., Penteado, P. F., See, C., Apr. 2012a. Radiative transfer analyses of Titan's tropical atmosphere. Icarus 
218, 975-988.

Griffith, C. A., Lora, J. M., Turner, J., Penteado, P. F., Brown, R. H., Tomasko, M. G., Doose, L., See, C., Jun. 2012b. Possible tropical lakes on Titan from observations of dark terrain. Nature 486, 237-239.

Griffith, C. A., Penteado, P., Baines, K., Drossart, P., Barnes, J., Bellucci, G., Bibring, J., Brown, R., Buratti, B., Capaccioni, F., Cerroni, P., Clark, R., Combes, M., Coradini, A., Cruikshank, D., Formisano, V., Jaumann, R., Langevin, Y., Matson, D., McCord, T., Mennella, V., Nelson, R., Nicholson, P., Sicardy, B., Sotin, C., Soderblom, L. A., Kursinski, R., Oct. 2005. The Evolution of Titan's Mid-Latitude Clouds. Science 310, 474-477.

Hayes, A., Aharonson, O., Callahan, P., Elachi, C., Gim, Y., Kirk, R., Lewis, K., Lopes, R., Lorenz, R., Lunine, J., Mitchell, K., Mitri, G., Stofan, E., Wall, S., May 2008. Hydrocarbon lakes on Titan: Distribution and interaction with a porous regolith. Geophysical Research Letters 35, L9204.

Hayes, A. G., Aharonson, O., Lunine, J. I., Kirk, R. L., Zebker, H. A., Wye, L. C., Lorenz, R. D., Turtle, E. P., Paillou, P., Mitri, G., Wall, S. D., Stofan, E. R., Mitchell, K. L., Elachi, C., Cassini Radar Team, Jan. 2011a. Transient surface liquid in Titan's polar regions from Cassini. Icarus 211, $655-671$.

Hayes, A. G., Aharonson, O., Lunine, J. I., Kirk, R. L., Zebker, H. A., Wye, L. C., Lorenz, R. D., Turtle, E. P., Paillou, P., Mitri, G., Wall, S. D., Stofan, E. R., Mitchell, K. L., Elachi, C., the Cassini RADAR Team, Jan. 
2011b. Transient surface liquid in Titan's polar regions from Cassini. Icarus $211,655-671$.

Hirtzig, M., Bézard, B., Lellouch, E., Coustenis, A., de Bergh, C., Drossart, P., Campargue, A., Boudon, V., Tyuterev, V., Rannou, P., Cours, T., Kassi, S., Nikitin, A., Mondelain, D., Rodriguez, S., Le Mouélic, S., Sep. 2013. Corrigendum to "Titan's surface and atmosphere from Cassini/VIMS data with updated methane opacity" [Icarus 226 (2013) 470-486]. Icarus $226,1182-1182$.

Hueso, R., Sánchez-Lavega, A., Jul. 2006. Methane storms on Saturn's moon Titan. Nature 442, 428-431.

Iess, L., Rappaport, N. J., Jacobson, R. A., Racioppa, P., Stevenson, D. J., Tortora, P., Armstrong, J. W., Asmar, S. W., Mar. 2010. Gravity Field, Shape, and Moment of Inertia of Titan. Science 327, 1367-.

Irwin, P. G. J., Lellouch, E., de Bergh, C., Courtin, R., Bézard, B., Fletcher, L. N., Orton, G. S., Teanby, N. A., Calcutt, S. B., Tice, D., Hurley, J., Davis, G. R., Jan. 2014. Line-by-line analysis of Neptune's near-IR spectrum observed with Gemini/NIFS and VLT/CRIRES. Icarus 227, 3748.

Jennings, D. E., Cottini, V., Nixon, C. A., Flasar, F. M., Kunde, V. G., Samuelson, R. E., Romani, P. N., Hesman, B. E., Carlson, R. C., Gorius, N. J. P., Coustenis, A., Tokano, T., 2011. Seasonal changes in titan's surface temperatures. The Astrophysical Journal Letters 737 (1), L15.

URL http://stacks.iop.org/2041-8205/737/i=1/a=L15 
Lacis, A. A., Oinas, V., May 1991. A description of the correlated-k distribution method for modelling nongray gaseous absorption, thermal emission, and multiple scattering in vertically inhomogeneous atmospheres. J. Geophys. Res. 96, 9027-9064.

Le Gall, A., Janssen, M. A., Wye, L. C., Hayes, A. G., Radebaugh, J., Savage, C., Zebker, H., Lorenz, R. D., Lunine, J. I., Kirk, R. L., Lopes, R. M. C., Wall, S., Callahan, P., Stofan, E. R., Farr, T., Jun. 2011. Cassini SAR, radiometry, scatterometry and altimetry observations of Titan's dune fields. Icarus 213, 608-624.

Lellouch, E., Bézard, B., Flasar, F. M., Vinatier, S., Achterberg, R., Nixon, C. A., Bjoraker, G. L., Gorius, N., Mar. 2014. The distribution of methane in Titan's stratosphere from Cassini/CIRS observations. Icarus 231, 323337.

Lora, J. M., Lunine, J. I., Russell, J. L., Hayes, A. G., Nov. 2014. Simulations of Titan's paleoclimate. Icarus 243, 264-273.

Lorenz, R. D., Aug. 2014. The flushing of Ligeia: Composition variations across Titan's seas in a simple hydrological model. Geophys. Res. Lett. 41, $5764-5770$.

Lorenz, R. D., Kirk, R. L., Hayes, A. G., Anderson, Y. Z., Lunine, J. I., Tokano, T., Turtle, E. P., Malaska, M. J., Soderblom, J. M., Lucas, A., Karatekin, Ö., Wall, S. D., Jul. 2014. A radar map of Titan Seas: Tidal dissipation and ocean mixing through the throat of Kraken. Icarus 237, 9-15. 
Lorenz, R. D., Mitchell, K. L., Kirk, R. L., Hayes, A. G., Aharonson, O., Zebker, H. A., Paillou, P., Radebaugh, J., Lunine, J. I., Janssen, M. A., Wall, S. D., Lopes, R. M., Stiles, B., Ostro, S., Mitri, G., Stofan, E. R., Jan. 2008. Titan's inventory of organic surface materials. Geophys. Res. Lett. $35,2206$.

Lorenz, R. D., Smith, P. H., Lemmon, M. T., May 2004. Seasonal change in Titan's haze 1992-2002 from Hubble Space Telescope observations. Geophys. Res. Lett. 31, 10702-+.

Lorenz, R. D., Stiles, B. W., Aharonson, O., Lucas, A., Hayes, A. G., Kirk, R. L., Zebker, H. A., Turtle, E. P., Neish, C. D., Stofan, E. R., Barnes, J. W., Jul. 2013. A global topographic map of Titan. Icarus 225, 367-377.

Lorenz, R. D., Wall, S., Radebaugh, J., Boubin, G., Reffet, E., Janssen, M., Stofan, E., Lopes, R., Kirk, R., Elachi, C., Lunine, J., Mitchell, K., Paganelli, F., Soderblom, L., Wood, C., Wye, L., Zebker, H., Anderson, Y., Ostro, S., Allison, M., Boehmer, R., Callahan, P., Encrenaz, P., Ori, G. G., Francescetti, G., Gim, Y., Hamilton, G., Hensley, S., Johnson, W., Kelleher, K., Muhleman, D., Picardi, G., Posa, F., Roth, L., Seu, R., Shaffer, S., Stiles, B., Vetrella, S., Flamini, E., West, R., May 2006. The Sand Seas of Titan: Cassini RADAR Observations of Longitudinal Dunes. Science 312, 724-727.

MacKenzie, S. M., Barnes, J. W., Sotin, C., Soderblom, J. M., Mouélic, S. L., Rodriguez, S., Baines, K. H., Buratti, B. J., Clark, R. N., Nicholson, P. D., McCord, T. B., 2014. Evidence of titan's climate history from evaporite distribution. Icarus 243, $191-207$. 
Mastrogiuseppe, M., Poggiali, V., Hayes, A., Lorenz, R., Lunine, J., Picardi, G., Seu, R., Flamini, E., Mitri, G., Notarnicola, C., Paillou, P., Zebker, H., 2014. The bathymetry of a titan sea. Geophysical Research Letters 41 (5), 1432-1437.

URL http://dx.doi.org/10.1002/2013GL058618

McLean, I. S., Becklin, E. E., Bendiksen, O., Brims, G., Canfield, J., Figer, D. F., Graham, J. R., Hare, J., Lacayanga, F., Larkin, J. E., Larson, S. B., Levenson, N., Magnone, N., Teplitz, H., Wong, W., Aug. 1998. Design and development of NIRSPEC: a near-infrared echelle spectrograph for the Keck II telescope. In: Fowler, A. M. (Ed.), Infrared Astronomical Instrumentation. Vol. 3354 of Society of Photo-Optical Instrumentation Engineers (SPIE) Conference Series. pp. 566-578.

Mitchell, J. L., Sep. 2012. Titan's Transport-driven Methane Cycle. ApJ 756, L26.

Mitchell, J. L., Ádámkovics, M., Caballero, R., Turtle, E. P., Sep. 2011. Locally enhanced precipitation organized by planetary-scale waves on Titan. Nature Geoscience 4, 589-592.

Mitchell, J. L., Pierrehumbert, R. T., Frierson, D. M. W., Caballero, R., Sep. 2009. The impact of methane thermodynamics on seasonal convection and circulation in a model Titan atmosphere. Icarus 203, 250-264.

Mitri, G., Showman, A. P., Lunine, J. I., Lorenz, R. D., Feb. 2007. Hydrocarbon lakes on Titan. Icarus 186, 385-394. 
Moore, J. M., Howard, A. D., Nov. 2010. Are the basins of Titan's Hotei Regio and Tui Regio sites of former low latitude seas? Geophys. Res. Lett. 37, L22205.

Niemann, H. B., Atreya, S. K., Bauer, S. J., Carignan, G. R., Demick, J. E., Frost, R. L., Gautier, D., Haberman, J. A., Harpold, D. N., Hunten, D. M., Israel, G., Lunine, J. I., Kasprzak, W. T., Owen, T. C., Paulkovich, M., Raulin, F., Raaen, E., Way, S. H., Dec. 2005. The abundances of constituents of Titan's atmosphere from the GCMS instrument on the Huygens probe. Nature 438, 779-784.

Niemann, H. B., Atreya, S. K., Demick, J. E., Gautier, D., Haberman, J. A., Harpold, D. N., Kasprzak, W. T., Lunine, J. I., Owen, T. C., Raulin, F., Dec. 2010. Composition of Titan's lower atmosphere and simple surface volatiles as measured by the Cassini-Huygens probe gas chromatograph mass spectrometer experiment. Journal of Geophysical Research (Planets) 115 (E14), 12006.

Penteado, P. F., Griffith, C. A., Mar. 2010. Ground-based measurements of the methane distribution on Titan. Icarus 206, 345-351.

Radebaugh, J., Lorenz, R. D., Lunine, J. I., Wall, S. D., Boubin, G., Reffet, E., Kirk, R. L., Lopes, R. M., Stofan, E. R., Soderblom, L., Allison, M., Janssen, M., Paillou, P., Callahan, P., Spencer, C., The Cassini Radar Team, Apr. 2008. Dunes on Titan observed by Cassini Radar. Icarus 194, 690-703. 
Rannou, P., Montmessin, F., Hourdin, F., Lebonnois, S., Jan. 2006. The Latitudinal Distribution of Clouds on Titan. Science 311, 201-205.

Rodriguez, S., Garcia, A., Lucas, A., Appéré, T., Le Gall, A., Reffet, E., Le Corre, L., Le Mouélic, S., Cornet, T., Courrech du Pont, S., Narteau, C., Bourgeois, O., Radebaugh, J., Arnold, K., Barnes, J. W., Stephan, K., Jaumann, R., Sotin, C., Brown, R. H., Lorenz, R. D., Turtle, E. P., Feb. 2014. Global mapping and characterization of Titan's dune fields with Cassini: Correlation between RADAR and VIMS observations. Icarus 230, 168-179.

Rodriguez, S., Le Mouélic, S., Rannou, P., Sotin, C., Brown, R. H., Barnes, J. W., Griffith, C. A., Burgalat, J., Baines, K. H., Buratti, B. J., Clark, R. N., Nicholson, P. D., Nov. 2011. Titan's cloud seasonal activity from winter to spring with Cassini/VIMS. Icarus 216, 89-110.

Rodriguez, S., Le Mouélic, S., Rannou, P., Tobie, G., Baines, K. H., Barnes, J. W., Griffith, C. A., Hirtzig, M., Pitman, K. M., Sotin, C., Brown, R. H., Buratti, B. J., Clark, R. N., Nicholson, P. D., Jun. 2009. Global circulation as the main source of cloud activity on Titan. Nature 459, 678-682.

Roe, H. G., May 2012. Titan's Methane Weather. Annual Review of Earth and Planetary Sciences 40, 355-382.

Roe, H. G., Brown, M. E., Schaller, E. L., Bouchez, A. H., Trujillo, C. A., Oct. 2005. Geographic Control of Titan's Mid-Latitude Clouds. Science $310,477-479$. 
Rothman, L. S., Gordon, I. E., Babikov, Y., Barbe, A., Chris Benner, D., Bernath, P. F., Birk, M., Bizzocchi, L., Boudon, V., Brown, L. R., Campargue, A., Chance, K., Cohen, E. A., Coudert, L. H., Devi, V. M., Drouin, B. J., Fayt, A., Flaud, J.-M., Gamache, R. R., Harrison, J. J., Hartmann, J.-M., Hill, C., Hodges, J. T., Jacquemart, D., Jolly, A., Lamouroux, J., Le Roy, R. J., Li, G., Long, D. A., Lyulin, O. M., Mackie, C. J., Massie, S. T., Mikhailenko, S., Müller, H. S. P., Naumenko, O. V., Nikitin, A. V., Orphal, J., Perevalov, V., Perrin, A., Polovtseva, E. R., Richard, C., Smith, M. A. H., Starikova, E., Sung, K., Tashkun, S., Tennyson, J., Toon, G. C., Tyuterev, V. G., Wagner, G., Nov. 2013. The HITRAN2012 molecular spectroscopic database. J. Quant. Spec. Radiat. Transf. 130, 4-50.

Schneider, T., Graves, S. D. B., Schaller, E. L., Brown, M. E., Jan. 2012. Polar methane accumulation and rainstorms on Titan from simulations of the methane cycle. Nature 481, 58-61.

Sotin, C., Lawrence, K. J., Reinhardt, B., Barnes, J. W., Brown, R. H., Hayes, A. G., Le Mouélic, S., Rodriguez, S., Soderblom, J. M., Soderblom, L. A., Baines, K. H., Buratti, B. J., Clark, R. N., Jaumann, R., Nicholson, P. D., Stephan, K., Nov. 2012. Observations of Titan's Northern lakes at $5 \mu \mathrm{m}$ : Implications for the organic cycle and geology. Icarus 221, 768-786.

Sromovsky, L. A., Fry, P. M., Boudon, V., Campargue, A., Nikitin, A., Mar. 2012. Comparison of line-by-line and band models of near-IR methane absorption applied to outer planet atmospheres. Icarus 218, 1-23.

Stamnes, K., Tsay, S.-C., Jayaweera, K., Wiscombe, W., 1988. Numerically 
stable algorithm for discrete-ordinate-method radiative transfer in multiple scattering and emitting layered media. Applied Optics 27, 2502-2509.

Stofan, E. R., Elachi, C., Lunine, J. I., Lorenz, R. D., Stiles, B., Mitchell, K. L., Ostro, S., Soderblom, L., Wood, C., Zebker, H., Wall, S., Janssen, M., Kirk, R., Lopes, R., Paganelli, F., Radebaugh, J., Wye, L., Anderson, Y., Allison, M., Boehmer, R., Callahan, P., Encrenaz, P., Flamini, E., Francescetti, G., Gim, Y., Hamilton, G., Hensley, S., Johnson, W. T. K., Kelleher, K., Muhleman, D., Paillou, P., Picardi, G., Posa, F., Roth, L., Seu, R., Shaffer, S., Vetrella, S., West, R., Jan. 2007. The lakes of Titan. Nature 445, 61-64.

Tokano, T., Mar. 2009. Limnological Structure of Titan's Hydrocarbon Lakes and Its Astrobiological Implication. Astrobiology 9, 147-164.

Tokano, T., Mar. 2014. Non-uniform global methane distribution in Titan's troposphere evidenced by Cassini radio occultations. Icarus 231, 1-12.

Tomasko, M. G., Doose, L., Engel, S., Dafoe, L. E., West, R., Lemmon, M., Karkoschka, E., See, C., Apr. 2008. A model of Titan's aerosols based on measurements made inside the atmosphere. Planet. Space Sci. 56, 669-707.

Turtle, E. P., Perry, J. E., Hayes, A. G., Lorenz, R. D., Barnes, J. W., McEwen, A. S., West, R. A., Del Genio, A. D., Barbara, J. M., Lunine, J. I., Schaller, E. L., Ray, T. L., Lopes, R. M. C., Stofan, E. R., Mar. 2011a. Rapid and Extensive Surface Changes Near Titan's Equator: Evidence of April Showers. Science 331, 1414-. 
Turtle, E. P., Perry, J. E., Hayes, A. G., McEwen, A. S., Apr. 2011b. Shoreline retreat at Titan's Ontario Lacus and Arrakis Planitia from Cassini Imaging Science Subsystem observations. Icarus 212, 957-959.

Turtle, E. P., Perry, J. E., McEwen, A. S., Del Genio, A. D., Barbara, J., West, R. A., Dawson, D. D., Porco, C. C., Jan. 2009. Cassini imaging of Titan's high-latitude lakes, clouds, and south-polar surface changes. Geophys. Res. Lett. 36 (2), L02204.

Vixie, G., Barnes, J. W., Jackson, B., Rodriguez, S., Le Mouélic, S., Wilson, P., 2014. Two Temperate Lakes on Titan. J. Geophys. Res., in revision. 
Table 1: Log of observations on 2014-07-17

\begin{tabular}{lccccc}
\hline \hline Target & Instrument & Time (UTC) & Exposure (s) & Airmass & Lon. $^{a}$ \\
\hline Titan & NIRSPAO & $05: 49: 30$ & 300 & 1.212 & 290.6 \\
Titan & NIRSPAO & $05: 55: 33$ & 300 & 1.214 & 290.7 \\
Titan & NIRSPAO & $06: 00: 47$ & 300 & 1.216 & 290.7 \\
Titan & NIRSPAO & $06: 07: 56$ & 300 & 1.220 & 290.9 \\
Titan & NIRSPAO & $06: 16: 28$ & 300 & 1.227 & 291.0 \\
Titan & NIRSPAO & $06: 23: 20$ & 300 & 1.234 & 291.1 \\
Titan & NIRSPAO & $06: 28: 34$ & 300 & 1.240 & 291.2 \\
Titan & NIRSPAO & $06: 34: 13$ & 300 & 1.248 & 291.3 \\
Titan & NIRSPAO & $06: 40: 19$ & 300 & 1.257 & 291.4 \\
Hip 77516 & NIRSPAO & $08: 30: 03$ & 20 & 1.254 & - \\
Titan & SINFONI & $23: 39: 31$ & 30 & 1.018 & 307.3 \\
Titan & SINFONI & $23: 40: 32$ & 30 & 1.018 & 307.4 \\
Titan & SINFONI & $23: 41: 31$ & 30 & 1.018 & 307.4 \\
Titan & SINFONI & $23: 42: 29$ & 30 & 1.018 & 307.4 \\
Hip 74680 & SINFONI & $23: 53: 21$ & 4 & 1.019 & - \\
\hline
\end{tabular}

${ }^{a}$ Sub-observer longitude from JPL Horizons ephemerides. 
Table 2: Atmospheric Structure ${ }^{a}$ and Composition ${ }^{b}$

\begin{tabular}{|c|c|c|c|c|c|c|c|}
\hline \multirow[t]{2}{*}{ Layer } & \multirow{2}{*}{$\begin{array}{c}\text { Pressure } \\
\text { (mbar) }\end{array}$} & \multirow{2}{*}{$\begin{array}{c}\text { Altitude } \\
(\mathrm{km})\end{array}$} & \multirow{2}{*}{$\begin{array}{c}\text { Temperature } \\
(\mathrm{K})\end{array}$} & \multirow{2}{*}{$\begin{array}{l}\text { Density } \\
\left(\mathrm{cm}^{-3}\right)\end{array}$} & \multicolumn{2}{|c|}{ Total Column } & \multirow{2}{*}{$\begin{array}{c}\mathrm{CH}_{4} \text { Column } \\
(\mathrm{km} \text { amg })\end{array}$} \\
\hline & & & & & $\left(\mathrm{cm}^{-2}\right)$ & (km amg) & \\
\hline 1 & 9.5 & 105.8 & 121.1 & $5.75 \mathrm{e}+17$ & $5.14 \mathrm{e}+24$ & 1.91 & 0.0286 \\
\hline 2 & 44.9 & 59.1 & 79.1 & $4.13 \mathrm{e}+18$ & $4.89 \mathrm{e}+24$ & 1.82 & 0.0272 \\
\hline 3 & 75.6 & 50.4 & 70.9 & $7.73 \mathrm{e}+18$ & $4.87 \mathrm{e}+24$ & 1.81 & 0.0272 \\
\hline 4 & 105.7 & 45.0 & 70.5 & $1.09 \mathrm{e}+19$ & $4.87 \mathrm{e}+24$ & 1.81 & 0.0272 \\
\hline 5 & 135.7 & 41.1 & 70.5 & $1.40 \mathrm{e}+19$ & $4.86 \mathrm{e}+24$ & 1.81 & 0.0275 \\
\hline 6 & 165.6 & 37.9 & 70.7 & $1.71 \mathrm{e}+19$ & $4.85 \mathrm{e}+24$ & 1.81 & 0.0282 \\
\hline 7 & 195.4 & 35.3 & 71.0 & $2.00 \mathrm{e}+19$ & $4.85 \mathrm{e}+24$ & 1.81 & 0.0293 \\
\hline 8 & 225.2 & 33.0 & 71.5 & $2.29 \mathrm{e}+19$ & $4.83 \mathrm{e}+24$ & 1.80 & 0.0302 \\
\hline 9 & 255.0 & 31.1 & 72.0 & $2.58 \mathrm{e}+19$ & $4.85 \mathrm{e}+24$ & 1.80 & 0.0313 \\
\hline 10 & 284.7 & 29.3 & 72.6 & $2.86 \mathrm{e}+19$ & $4.84 \mathrm{e}+24$ & 1.80 & 0.0323 \\
\hline 11 & 353.5 & 25.7 & 74.1 & $3.49 \mathrm{e}+19$ & $1.90 \mathrm{e}+25$ & 7.07 & 0.1398 \\
\hline 12 & 471.4 & 20.9 & 76.6 & $4.51 \mathrm{e}+19$ & $1.89 \mathrm{e}+25$ & 7.05 & 0.1665 \\
\hline 13 & 588.7 & 17.1 & 78.9 & $5.47 \mathrm{e}+19$ & $1.88 \mathrm{e}+25$ & 7.00 & 0.1942 \\
\hline 14 & 705.9 & 13.9 & 81.1 & $6.38 \mathrm{e}+19$ & $1.89 \mathrm{e}+25$ & 7.02 & 0.2350 \\
\hline 15 & 822.8 & 11.1 & 83.1 & $7.25 \mathrm{e}+19$ & $1.88 \mathrm{e}+25$ & 7.00 & 0.2831 \\
\hline 16 & 939.7 & 8.7 & 84.9 & $8.10 \mathrm{e}+19$ & $1.87 \mathrm{e}+25$ & 6.96 & 0.3363 \\
\hline 17 & 1056.6 & 6.5 & 86.8 & $8.92 \mathrm{e}+19$ & $1.87 \mathrm{e}+25$ & 6.98 & 0.3754 \\
\hline 18 & 1173.4 & 4.5 & 88.7 & $9.72 \mathrm{e}+19$ & $1.88 \mathrm{e}+25$ & 7.01 & 0.4002 \\
\hline 19 & 1290.2 & 2.6 & 90.6 & $1.05 \mathrm{e}+20$ & $1.88 \mathrm{e}+25$ & 7.01 & 0.4027 \\
\hline 20 & 1406.9 & 0.8 & 92.5 & $1.12 \mathrm{e}+20$ & $1.89 \mathrm{e}+25$ & 7.04 & 0.3514 \\
\hline
\end{tabular}


Table 3: Comparison of aerosol models used for SINFONI observations

\begin{tabular}{lcl}
\hline \hline Model ID & $\hat{\chi}^{2}$ & Note \\
\hline DISR & 4.72 & Tomasko et al. (2008) at all latitudes. \\
$P(\theta)$ & 3.61 & No back-scattering in phase function. \\
$P(\theta), \omega_{H}=0.94$ & 2.86 & No back-scattering, lower scattering albedo. \\
$f_{H}=0.5$ & 1.02 & Scaled DISR aerosol at all latitudes. \\
LM 2-parameter & 0.68 & Variable haze above 10 km. \\
LM 3-parameter & 0.57 & Variable haze to surface, and above $65 \mathrm{~km}$. \\
\hline
\end{tabular}




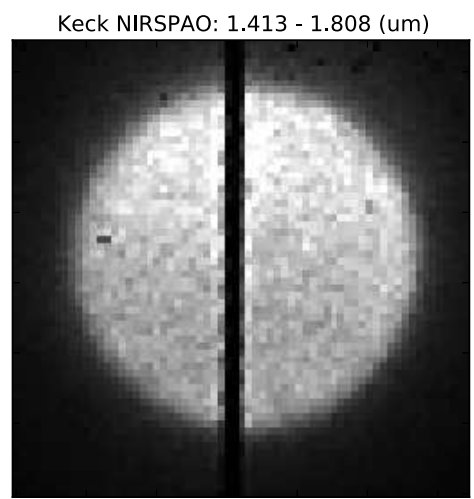

VLT SINFONI: $1.577-1.597(\mathrm{um})$

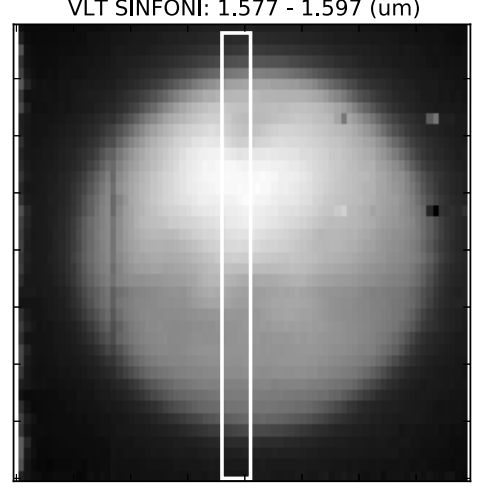

2014-07-17 UT

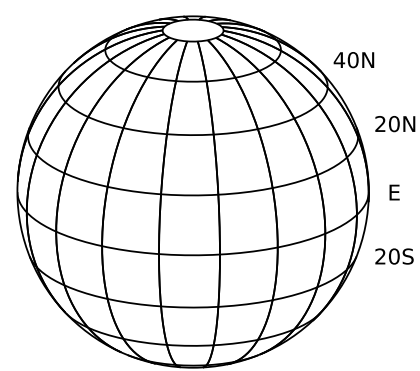

lat $\odot$, lon $_{\odot}=22.6 \mathrm{~N}, 301 \mathrm{~W}$

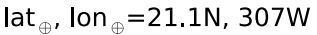

Cassini VIMS: 1.552 (um)

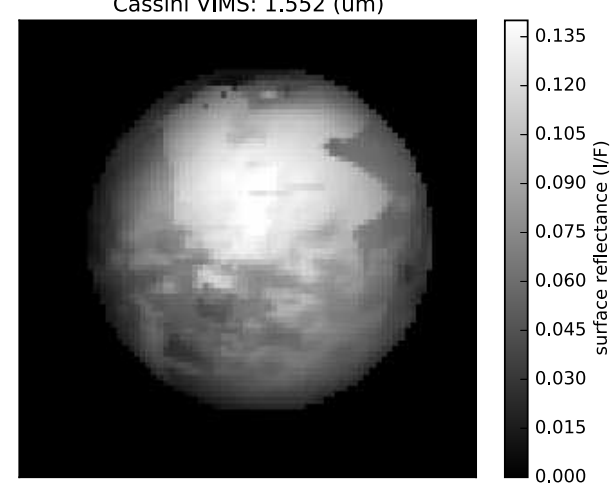

Figure 1: Viewing geometry and surface albedo of Titan during observations. An H-band image from the NIRSPAO slit viewing camera (top left) during the spectrometer exposures identifies the spatial location of the spectra. The wide bandpass results in sensitivity to the atmospheric haze. An image from the SINFONI data cube (bottom left) that is sensitive to surface albedo variations is shown with a white box that indicates the region of pixels that corresponds to the NIRSPAO slit. This is the region considered when referring to pixels along the SINFONI "slit". An orthographic reprojection of a $1.552 \mu \mathrm{m}$ Cassini Visual and Infrared Mapping Spectrometer (VIMS) map, sampled at the SINFONI plate scale and artificially limb-darkened, illustrates the surface reflectance in the absence of the atmospheric contribution and confirms the photometry and flux calibration of the SINFONI data (bottom right). The viewing geometry of the observations is show in the schematic (top right) with a grid spacing of 20 degrees. 


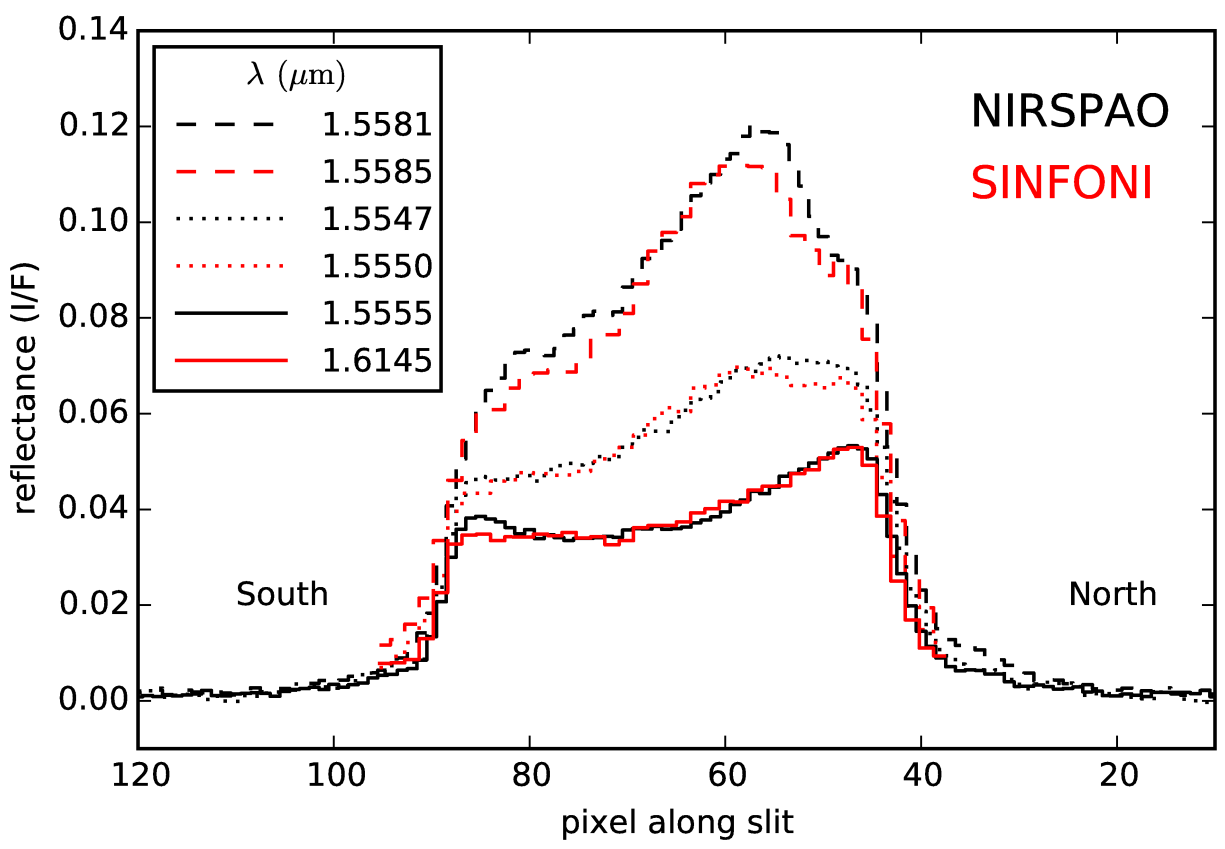

Figure 2: Reflectance profiles along the NIRSPAO slit (black) and corresponding SINFONI pixels (red) at wavelengths that are sensitive to the surface (dashed), and atmosphere (dotted and solid). The profiles that are sensitive to the surface are limb-darkened whereas the profiles sensitive to the atmosphere exhibit limb-brightening. The spatial region of pixels that is used from the SINFONI cube is illustrated by the white box in the bottom left panel of Figure 1. The SINFONI pixel scale has been linearly mapped to the NIRSPAO pixel scale. The difference between reflectivity profiles of the surface (red and black dashed curves) is consistent with the surface albedo changing due to the rotation between the time of the two observations. 

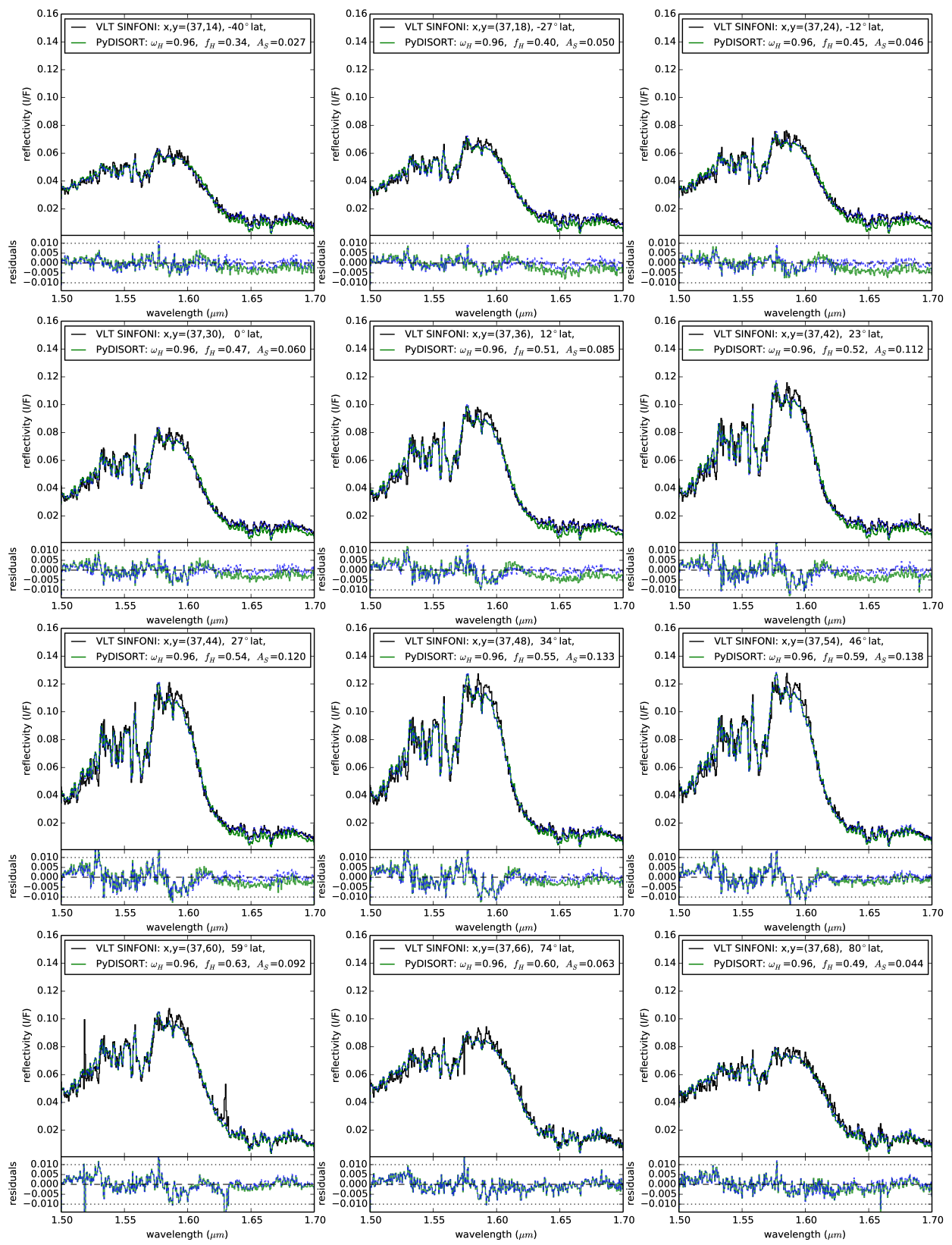

Figure 3: VLT SINFONI spectra (black) with best fit 2-parameter models (green) at 12 locations in the SINFONI FOV corresponding to the NIRSPAO slit. The free parameters in the model are the surface albedo, $A_{S}$, and a scale factor, $f_{H}$, for the atmospheric haze, which is known to vary spatially and temporally relative to the haze structure measured by the DISR. A 3-parameter model fit (blue), where scale factors for hazes above and below $65 \mathrm{~km}$ are two separate free parameters, produces a comparable fit to the observations from $1.50-1.62 \mu \mathrm{m}$, while fitting the observations better from $1.63-1.70 \mu \mathrm{m}$. 


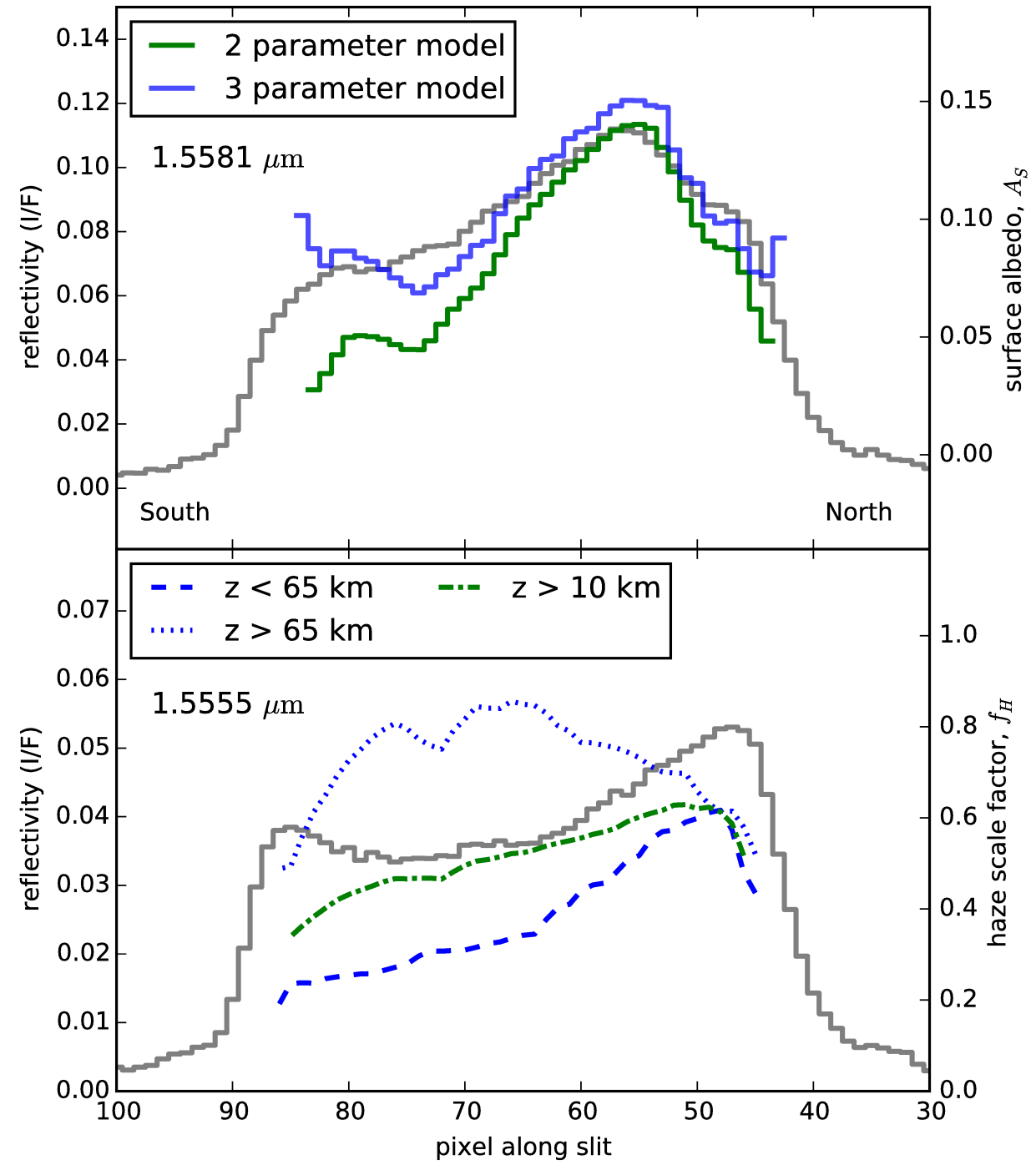

Figure 4: Parameters from LM optimization of model spectra fit to SINFONI observations along the slit (a subset are shown in Figure 3), together with the NIRSPAO reflectivity at individual wavelengths (black) that are sensitive to these parameters. The best fit surface albedos (top), where $A_{S}$ is always one of the free parameters in the 2 and 3 parameters models (solid green and blue, respectively). And the haze scale factors, $f_{H}$, (bottom panel) that are used for either the entire vertical profile above $10 \mathrm{~km}$ (green dot-dash curve) or as two separate free parameters for the hazes above (blue dotted) and below $65 \mathrm{~km}$ (blue dashed). The 3-parameter model has a larger gradient in lower atmospheric haze than the 2-parameter model, and the stratospheric hazes decrease toward both poles. The large haze gradient in the 3-parameter model is balanced by a small gradient in the surface albedo. The model parameters have been interpolated onto the NIRSPAO grid and are used as input parameters for fitting the NIRSPAO spectra. 

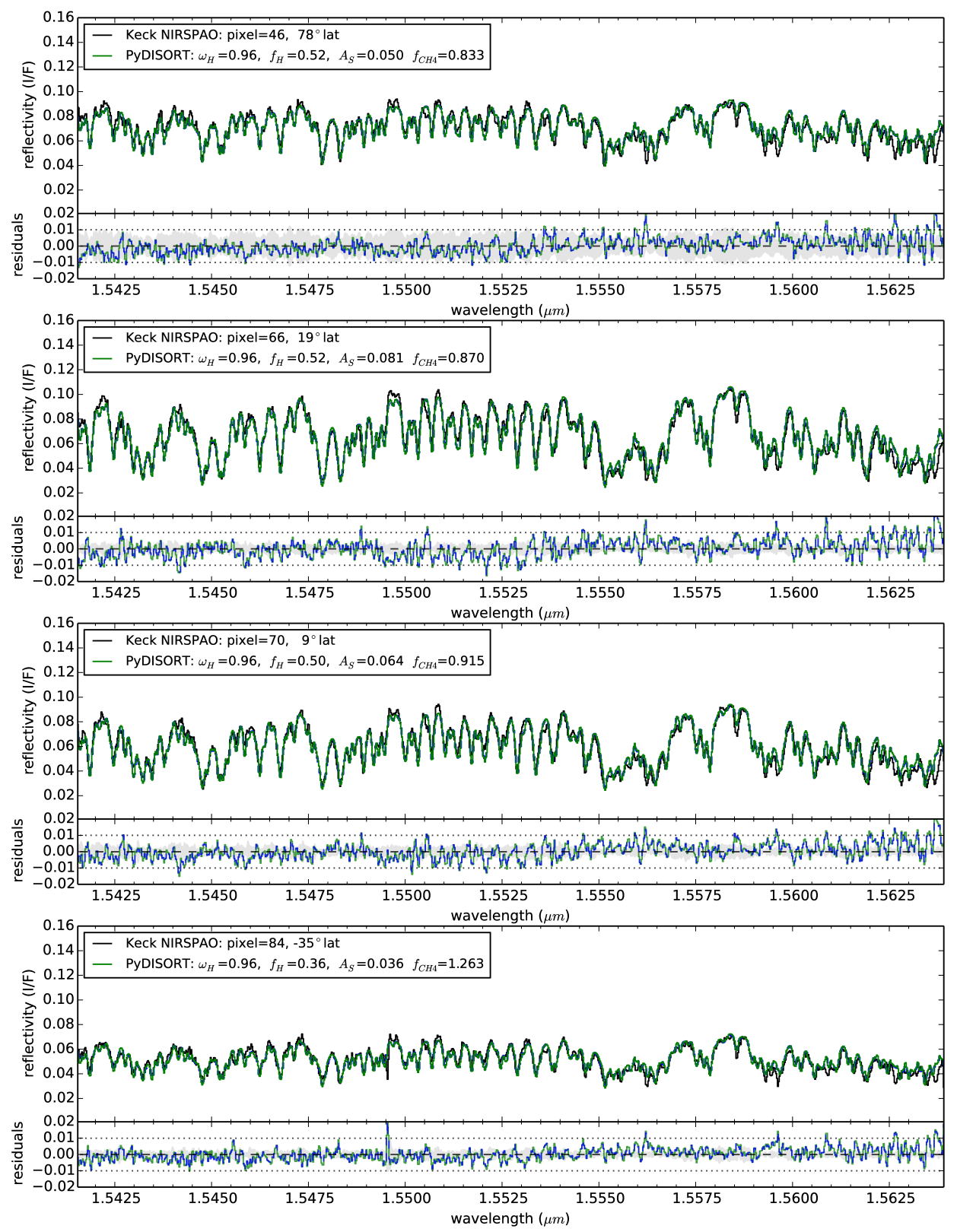

Figure 5: Keck NIRSPAO spectra from four individual pixels along the slit (black) with models from a Levenberg-Marquardt optimization using inputs from either 2-parameter (green) or 3-parameter (blue) fitting of SINFONI spectra. The 2-parameter models assume uniform troposphere haze and only vary the aerosol above $10 \mathrm{~km}$, whereas the 3 -parameter models are optimized to the observations with a significant gradient in tropospheric aerosol (Figure 4 ). The free parameters when fitting the NIRSPAO spectra are $f_{\mathrm{CH} 4}, \delta A_{S}$, and $f_{H}^{\prime}$ (see text for details). These four spectra are a subset of the 42 pixels that sample the disk of Titan and are used for methane abundance retrievals. 


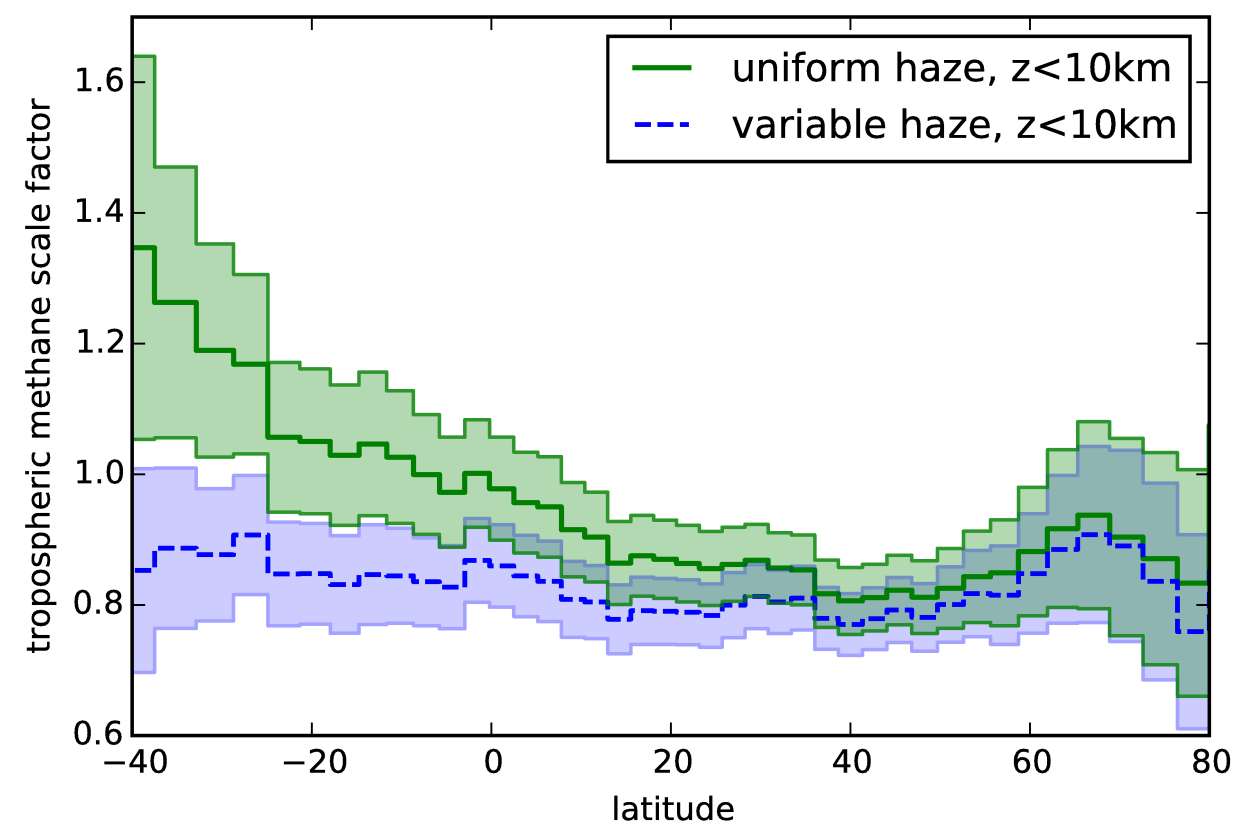

Figure 6: Meridional variation in the tropospheric methane abundance relative to the mole fraction measured by the GCMS on the Huygens probe retrieved from fits to NIRSPAO spectra. Examples spectra and best-fit models are illustrated in Figure 5. 

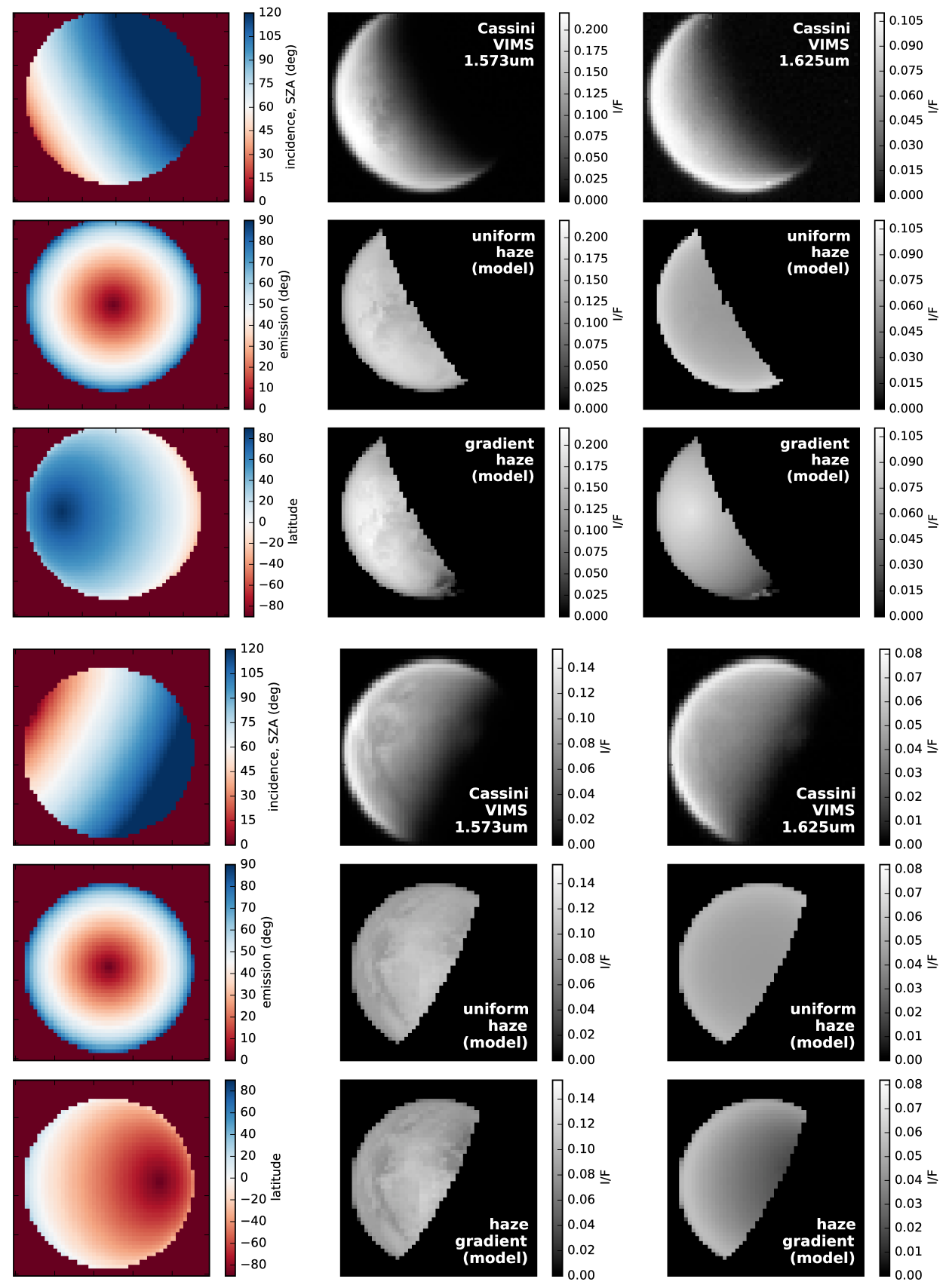

Figure 7: Cassini VIMS images and models (grayscale) of the surface and near-surface hazes in the northern hemisphere during the T103 flyby on 2014 Jul 20 (egress). Viewing geometry is illustrated in the color panels, left column. The synthetic images are calculated with aerosol models that assume either a ufform haze at all latitudes, or a models with a haze gradient that increases $f_{H}$ with latitude toward the North. 